\title{
An algebraic model for the propagation of errors in matrix calculus
}

https://doi.org/10.1515/spma-2020-0008

Received November 18, 2019; accepted February 12, 2020

\begin{abstract}
We assume that every element of a matrix has a small, individual error, and model it by an external number, which is the sum of a nonstandard real number and a neutrix, the latter being a convex (external) additive group. The algebraic properties of external numbers formalize common error analysis, with rules for calculation which are a sort of mellowed form of the axioms for real numbers.

We model the propagation of errors in matrix calculus by the calculus of matrices with external numbers, and study its algebraic properties. Many classical properties continue to hold, sometimes stated in terms of inclusion instead of equality. There are notable exceptions, for which we give counterexamples and investigate suitable adaptations. In particular we study addition and multiplication of matrices, determinants, near inverses, and generalized notions of linear independence and rank.
\end{abstract}

Keywords: matrix calculus; error propagation; rank; external numbers.

MSC: 03H05, 15A03, 15A09; 15B33, 65F99.

\section{Introduction}

In this article imprecisions in entries of matrices are modelled by (scalar) neutrices, which are convex subgroups of the set of nonstandard real numbers, most of them are external sets. They are a sort of generalized zeros. Each entry of a matrix is an external number, which is the pointwise (Minkowski) sum of a (nonstandard) real number and a neutrix. Every entry has its own individual neutrix, modelling the diversity of imprecisions. The intrinsic vagueness is respected by the Sorites property of neutrices, which are invariant by some shifts. Examples of neutrices are the external set of infinitesimals $\oslash$ and the external set $€$ of numbers smaller in absolute value than some standard real number, as well as all multiples of them, but there exist other types of neutrices [25]. The term neutrix is borrowed from Van der Corput, and we were inspired by his Ars Negligendi [5].

Within the setting of external numbers we study the effects of error propagation in calculations with matrices and determinants.

The calculus of external numbers originates from error analysis, which is more or less informal. "Provisional" rules for addition, subtraction, multiplication and division are for instance given in [35], and they lead only to a weak algebraic structure. In the context of external numbers these rules are formalized as Minkowski operations. The fact that neutrices are convex additive groups enables us to build a much stronger algebraic structure, called Complete Arithmetical Solid in [11]. Addition and multiplication satisfy the properties of a completely regular commutative semigroup [32], and adapted forms of distributivity, order relation, Dedekind completeness and the Archimedean property are shown to hold.

\footnotetext{
`Corresponding Author: Nam Van Tran: Faculty of Applied Sciences, HCMC University of Technology and Education, Vietnam, E-mail: namtv@hcmute.edu.vn

Imme van den Berg: Research Center in Mathematics and Applications, University of Évora, Portugal, E-mail: ivdb@uevora.pt
} 
We cannot hope that such strong rules hold for matrix calculus, still the matrices form a regular commutative semigroup for addition: the usual laws for addition are valid, but the sum of a matrix and its additive "inverse" will be a matrix of neutrices, and not the zero-matrix. Also in many cases the common laws for multiplication of matrices hold. Problems may appear when multiplying matrices with entries of different sign, in particular when some entries are almost equal in absolute value but opposite, or when the matrix has a small determinant. Still many algebraic properties hold under quite general conditions, typically entries should not be nearly opposite, a notion defined in Section 2. Sometimes algebraic properties hold in the form of inclusions instead of equalities.

We pay special attention to invertibility, linear dependence and independence, and rank.

In analogy to addition, generically we cannot hope that the product of two matrices yields the identity matrix. We speak of a near inverse if we obtain the identity matrix up to neutrices included in $\oslash$. We give conditions for near inverses to exist, in terms of not too small determinants.

We give a straightforward definition for linear independence of vectors of external numbers, and relate it to classical linear independence and dependence of vectors of representatives, i.e. real numbers which are elements of the external numbers.

There are several notions of rank of a matrix of external numbers. The row rank s is defined in the common way, using linear independence. The minor rank is defined using the non-singularity of minors. In fact a mixed notion called strict rank happens to be the more operational. We give conditions for its existence, and show that then the row rank is equal to the minor rank.

This article has the following structure. In Section 2 we present some properties of neutrices and external numbers, which are needed for the remaining sections. Some results are recalled, some are new. In Section 3 we show that almost all common properties of operations on matrices hold for non-negative matrices, and give general conditions for these properties to hold beyond. Section 4 deals with the determinant and its minors. In Section 5 we study nearly invertible matrices. In Section 6 we extend the notions of linear dependence and independence to external vectors. Section 7 discusses several notions of rank and their relationships. In Section 8 we relate briefly our approach to other forms of dealing with imprecisions and errors.

\section{Neutrices and external numbers}

We recall the definitions of neutrices and external numbers, and some basic properties as regards to algebraic rules and the order relation. We derive some new properties which are useful to matrix calculus. For more details on neutrices and external numbers we refer to [2, 10-12, 25].

Remark 2.1. Throughout this article we use the symbol $\subseteq$ for inclusion and $\subset$ for strict inclusion.

Neutrices and external numbers are well-defined external sets in the axiomatic system HST for nonstandard analysis as given by Kanovei and Reeken in [24]. This is an extension of a bounded form of Nelson's Internal Set Theory IST [29]. This theory extends common set theory ZFC by adding an undefined predicate "standard" to the language of set theory, and three new Axiom schemes. Introductions to IST are contained in e.g. [9], [8] or [26]. An important feature is that infinite sets always have nonstandard elements. In particular nonstandard numbers are already present within $\mathbb{R}$. Limited numbers are real numbers bounded in absolute value by standard natural numbers. Real numbers larger in absolute value than limited numbers are called unlimited. Its reciprocals, together with 0 , are called infinitesimal. Limited numbers which are not infinitesimal are called appreciable.

A (scalar) neutrix is an additive convex subgroup of $\mathbb{R}$. Except for $\{0\}$ and $\mathbb{R}$, all neutrices are external sets. The set of all limited numbers $€$ and the set of all infinitesimals $\oslash$ are neutrices. Note that $€$ and $\oslash$ are not sets in the sense of $Z F C$, for they are bounded subsets of $\mathbb{R}$ with no lowest upper bound. Let $\varepsilon \in \mathbb{R}$ be a positive infinitesimal. Some other neutrices are $\varepsilon \oslash, \varepsilon £, \bigcap_{s t(n) \in \mathbb{N}}\left[-\varepsilon^{n}, \varepsilon^{n}\right]=£ \varepsilon^{\infty}, \bigcup_{s t(n) \in \mathbb{N}}\left[-e^{-1 /(n \varepsilon)}, e^{-1 /(n \varepsilon)}\right]=£ e^{-@ / \varepsilon}$; here 
@ denotes the external set of positive appreciable numbers and $\varnothing$ the external set of positive unlimited numbers. For every neutrix $N$ it holds that $€ N=N$.

An external number is the Minkowski-sum of a real number and a neutrix. So each external number has the form $\alpha=a+A=\{a+x \mid x \in A\}$, where $A$ is called the neutrix part of $\alpha$, denoted by $N(\alpha)$, and $a \in \mathbb{R}$ is called a representative of $\alpha$. If $N(\alpha)=\{0\}$, we may identify $\{a\}$ and $a$, so that the real numbers are external numbers. If $0 \notin \alpha=a+N(\alpha)$, we call $\alpha$ zeroless and then

$$
\alpha \cap \oslash \alpha=\emptyset .
$$

Sometimes we call $\alpha$ neutricial if $\alpha=N(\alpha)$.

The collection of all neutrices is not an external set, but a definable class, denoted by $\mathcal{N}$. Also the external numbers form a class, denoted by $\mathbb{E}$.

Addition, subtraction, multiplication and division are given by the Minkowski operations of Definition 2.2 below.

Definition 2.2. Let $\alpha=a+A, \beta=b+B$ be two external numbers and $A, B$ be two neutrices.

1. $\alpha \pm \beta=a \pm b+A+B=a+b+\max \{A, B\}$.

2. $\alpha \beta=a b+A b+B a+A B=a b+\max \{a B, b A, A B\}$.

3. If $\alpha$ is zeroless, $\frac{1}{\alpha}=\frac{1}{a}+\frac{A}{a^{2}}$.

Neutrices are ordered by inclusion, and the maximums are taken in this sense. If $\alpha$ or $\beta$ are zeroless, in Definition 2.2.2 we may neglect the neutrix product $A B$.

The rules of Definition 2.2 reflect the common rules for the propagation of errors of error analysis. In [35] they are called "provisional rules", for this analysis is informal, to hold approximately and somewhat ad hoc, using common sense. In contrast, in terms of external numbers, the equalities of Definition 2.2 are part of formal mathematics and permit us to prove much more general laws, which lead to the notion of Complete Arithmetical Solid in [11]. This structure is a completely regular commutative semigroup [32] for addition and multiplication, and distributivity, the order relation, Dedekind completeness and the Archimedean property hold in modified forms.

We consider here only some properties which are useful for the remainder of this article. We recall some properties of neutrices and the order relation, and give some special attention to distributivity, which is of importance for the matrix calculus.

Definition 2.3. Let $N$ be a neutrix and $\alpha$ be an external number. The external number $\alpha$ is called an absorber of $N$ if $\alpha N \subset N$, and an exploder of $N$ if $N \subset \alpha N$.

We have $t A=A$ for all $|t| \in @$, so appreciables are neither absorbers nor exploders of a neutrix $A$. Infinitesimals are absorbers of $€$ and $\oslash$, and unlimited numbers are exploders of these neutrices. Observe that if $\varepsilon \in \mathbb{R}$ is a positive infinitesimal, it is not an absorber of $\epsilon \varepsilon^{\infty}$, nor of $€ e^{-@ / \varepsilon}$, and its reciprocal $1 / \varepsilon$ is not an exploder for these neutrices.

Definition 2.2.3 does not permit to divide by neutrices. However we will use the common notation for division of groups.

Definition 2.4. Let $A, B \in \mathcal{N}$. Then we define

$$
A: B=\{c \in \mathbb{R} \mid c B \subseteq A\} .
$$

An order relation for all external numbers $\alpha, \beta$ is given by

$$
\alpha \leq \beta \equiv \forall a \in \alpha \exists b \in \beta(a \leq b) .
$$

If $\alpha \cap \beta=\emptyset$ and $\alpha \leq \beta$, then $\forall a \in \alpha \forall b \in \beta(a<b)$ and we write $\alpha<\beta$. Note that $\oslash \supsetneqq E$, while $\oslash \cap £ \neq \emptyset$, so $\oslash \nless €$. An external number $\alpha$ is called positive if $0<\alpha$ and negative if $\alpha<0$. The number $\alpha$ is non-negative if 
$0 \leq \alpha$, i.e. if there exists $x \in \alpha$ such that $0 \leq x$ and non-positive if $0 \geq \alpha$; this means that there exists $x \in \alpha$ with $0 \geq x$. Note that a neutrix is both non-negative and non-positive. The class of all non-negative external numbers will be denoted by $\mathbb{E}^{+}$. The order relation is shown to be compatible with the operations, with some small adaptations [12, 25].

Definition 2.5. Let $\alpha=a+A$ be an external number. The absolute value of $\alpha$ is defined by $|\alpha|=|a|+A$.

Notice that this definition does not depend on the choice of the representative of $\alpha$.

In the final part of this section we consider the modified distributivity law. It takes the following form.

Theorem 2.6. $[10]$ (Distributivity with correction term) Let $\alpha, \beta, \gamma=c+C$ be extermal numbers. Then

$$
\alpha \gamma+\beta \gamma=(\alpha+\beta) \gamma+C \alpha+C \beta .
$$

Because a neutrix term is added in the right-hand side of (2), we always have the following form of subdistributivity.

Corollary 2.7. (Subdistributivity) Let $\alpha, \beta$, $\gamma$ be external numbers. Then $(\alpha+\beta) \gamma \subseteq \alpha \gamma+\beta \gamma$.

Full distributivity holds under some conditions, see Theorem 2.10 below. The conditions are formulated with the help of the notions of relative uncertainty and oppositeness. Definition 2.8 is from [10], which contains illustrative examples.

Definition 2.8. Let $\alpha=a+A$ and $\beta=b+B$ be external numbers and $C$ be a neutrix.

1. The relative uncertainty $R(\alpha)$ of $\alpha$ is defined by $A / \alpha$ if $\alpha$ is zeroless, otherwise $R(\alpha)=\mathbb{R}$.

2. $\alpha$ and $\beta$ are opposite with respect to $C$ if $(\alpha+\beta) C \subset \max (\alpha C, \beta C)$.

In Definition 2.8.2 the external number $(\alpha+\beta) /(\max (|\alpha|,|\beta|)$ must be so small to be an absorber of $C$, so $\alpha$ and $\beta$ should indeed wipe each other almost out.

Proposition 2.9. $[10,25]$ Let $\alpha=a+A$ be zeroless. Then:

1. $\alpha N=a N$ and $N / \alpha=N / a$.

2. $R(\alpha)=A / a \subseteq \oslash$.

3. $R(1 / \alpha)=R(\alpha)$.

4. $\alpha=\alpha(1+R(\alpha))=a(1+R(\alpha))$.

We now are able to formulate the criteria for distributivity.

Theorem 2.10. [10] Let $\alpha, \beta, \gamma=c+C$ be external numbers. Then $\alpha \gamma+\beta \gamma=(\alpha+\beta) \gamma$ if and only if $R(\gamma) \subseteq$ $\max (R(\alpha), R(\beta))$, or $\alpha$ and $\beta$ are not opposite with respect to $C$.

Obviously distributivity holds if $\alpha$ and $\beta$ are of the same sign, say if we are always working with positive numbers or non-negative numbers.

We end this section with three propositions on relative precision and distributivity, which are useful in the study of matrices with external numbers.

Proposition 2.11. Let $n \in \mathbb{N}$ be standard and $\alpha_{1}, \ldots, \alpha_{n}$ be external numbers. Let $\lambda=\alpha_{1} \cdots \alpha_{n}$. Then $R(\lambda)=$ $\sum_{i=1}^{n} R\left(\alpha_{i}\right)=\max _{1 \leq i \leq n} R\left(\alpha_{i}\right)$. 
Proof. For $1 \leq i \leq n$, let $\alpha_{i}=a_{i}+A_{i}$. Obviously $\sum_{i=1}^{n} R\left(\alpha_{i}\right)=\max _{1 \leq i \leq n} R\left(\alpha_{i}\right)$. If $\max _{1 \leq i \leq n} R\left(\alpha_{i}\right)=\mathbb{R}$, there exists $i_{0} \in$ $\{1, \ldots, n\}$ such that $\alpha_{i_{0}}$ is a neutrix. It follows that $\lambda$ is a neutrix. Hence $R(\lambda)=\mathbb{R}=\max _{1 \leq i \leq n} R\left(\alpha_{i}\right)$. Otherwise, let $2 \leq p \leq n, i_{1}, \ldots, i_{p} \in\{1, \ldots, n\}$ and $J=\{1, \ldots, n\} \backslash\left\{i_{1}, \ldots, i_{p}\right\}$. Then

$$
\begin{aligned}
\lambda & =a_{1} \ldots a_{n}+\sum_{p=1}^{n} \sum_{1 \leq i_{1}<\ldots<i_{p} \leq n}\left(A_{i_{1}} \ldots A_{i_{p}} \Pi_{j \in J} a_{j}\right) \\
& =a_{1} \ldots a_{n}+A_{1} a_{2} \ldots a_{n}+\cdots+A_{n} a_{1} \ldots a_{n-1} \\
& +\sum_{p=2}^{n} \sum_{1 \leq i_{1}<\cdots<i_{p} \leq n}\left(A_{i_{1}} \ldots A_{i_{p}} \Pi_{j \in J} a_{j}\right) .
\end{aligned}
$$

Put

$$
\mu_{p}=a_{1} \cdots a_{n}+\sum_{1 \leq i_{1}<\cdots<i_{p} \leq n}\left(A_{i_{1}} \ldots A_{i_{p}} \Pi_{j \in J} a_{j}\right) .
$$

Then

$$
R(\lambda)=\sum_{i=1}^{n} R\left(\alpha_{i}\right)+\sum_{p=2}^{n} R\left(\mu_{p}\right)=\max _{1 \leq i \leq n} R\left(\alpha_{i}\right)+\sum_{p=2}^{n} R\left(\mu_{p}\right) .
$$

By Proposition 2.9.2 we have $R\left(\alpha_{i}\right) \subseteq \oslash$ for $1 \leq i \leq n$. This implies that $R\left(\alpha_{i_{1}}\right) \cdots R\left(\alpha_{i_{p}}\right) \leq \max _{1 \leq i \leq n} R\left(\alpha_{i}\right)$. As a result $R\left(\mu_{p}\right) \leq \max _{1 \leq i \leq n} R\left(\alpha_{i}\right)$.

So $\sum_{p=2}^{n} R\left(\mu_{p}\right) \leq \sum_{p=2}^{n} \max _{1 \leq i \leq n} R\left(\alpha_{i}\right)=\max _{1 \leq i \leq n} R\left(\alpha_{i}\right)$. Hence $R(\lambda)=\max _{1 \leq i \leq n} R\left(\alpha_{i}\right)=\sum_{i=1}^{n} R\left(\alpha_{i}\right)$.

It follows from Proposition 2.11 that whenever $\alpha, \beta \in \mathbb{E}$, it holds that $R(\alpha \beta)=R(\alpha)+R(\beta)=\max (R(\alpha), R(\beta)$ ), and in case $\beta$ is zeroless, by Proposition 2.9.3 also $R\left(\frac{\alpha}{\beta}\right)=R(\alpha)+R\left(\frac{1}{\beta}\right)=R(\alpha)+R(\beta)=\max (R(\alpha), R(\beta))$.

Proposition 2.12. Let $n \in \mathbb{N}$ be standard, $\alpha, \beta_{1}, \ldots, \beta_{n}$ be external numbers. If $R(\alpha) \leq \min _{1 \leq i \leq n} R\left(\beta_{i}\right)$, then $\alpha\left(\beta_{1}+\right.$ $\left.\cdots+\beta_{n}\right)=\alpha \beta_{1}+\cdots+\alpha \beta_{n}$.

Proof. For $n=2$ the equality holds by Theorem 2.10. We will apply external induction. Let $k$ be standard, and suppose the equality holds for $n=k$. Let $\beta=\beta_{1}+\cdots+\beta_{k}$. Then $\alpha\left(\beta_{1}+\cdots+\beta_{k+1}\right)=\alpha\left(\beta+\beta_{k+1}\right)=\alpha \beta+\alpha \beta_{k+1}=$ $\alpha \beta_{1}+\cdots+\alpha \beta_{k}+\alpha \beta_{k+1}$. By external induction we conclude that the equality holds for every standard natural number $n$.

By Theorem 2.10 distributivity certainly holds with respect to external numbers of the same sign, but we may weaken this to nearly opposite numbers, as given by the next definition.

Definition 2.13. Two zeroless elements $\alpha, \beta \in \mathbb{E}$ are nearly opposite if $\alpha / \beta \subseteq-1+\oslash$.

For example, a real number $b \simeq 1$ and -1 are nearly opposite, but they are not opposite with respect to 0 . If $a$ and $b$ are two standard real numbers such that $b \neq-a$, they are not nearly opposite.

Proposition 2.14. Let $\alpha, \beta, \gamma \in \mathbb{E}$ be such that $\alpha$ and $\beta$ are not nearly opposite. Then $(\alpha+\beta) \gamma=\alpha \gamma+\beta \gamma$.

Proof. Let $\gamma=c+C$. The distributive law holds if $\alpha$ or $\beta$ is neutricial. In case both are zeroless, we may suppose that $|\alpha| \leq|\beta|$. Then with $\beta=b+B$ we have $\left|\frac{\alpha}{b}\right| \leq 1+\oslash$. Also $\alpha$ and $b$ are not nearly opposite, so $1+\frac{\alpha}{b} \subset @$, hence by Proposition 2.9.2 also $1+R(\beta)+\frac{\alpha}{b}$ is a subset of @. Then $1+R(\beta)+\frac{\alpha}{b}$ is neither an absorber, nor an exploder of $C$. Then by Theorem 2.10 and Proposition 2.9.1.

$$
(\alpha+\beta) C=b\left(1+R(\beta)+\frac{\alpha}{b}\right) C=b C=\beta C=\max (\alpha C, \beta C)=\alpha C+\beta C .
$$


Hence by Theorem 2.10 and Theorem 2.6

$$
\begin{aligned}
(\alpha+\beta) \gamma & =(\alpha+\beta)(c+C)=(\alpha+\beta) C+(\alpha+\beta) C \\
= & (\alpha+\beta) C+(\alpha+\beta) C+(\alpha+\beta) C=(\alpha+\beta) \gamma+\alpha C+\beta C=\alpha \beta+\alpha \gamma .
\end{aligned}
$$

\section{Matrices with external numbers}

In this section operations on matrices with external numbers are studied. We start with addition, and show that it satisfies the rules of a regular commutative semigroup. Then we study scalar multiplication and matrix multiplication. In many cases, in particular if the elements of the matrices are of the same sign, the same laws hold as for real matrices. External numbers satisfy the subdistributivity property, and the same is true for scalar multiplication and matrix multiplication. We present conditions for the distributivity property to hold. In contrast to the multiplication of external numbers, the associative property does not hold for scalar multiplication and matrix multiplication. We provide conditions so that the subassociativity property is valid, and conditions so that the associativity property is valid.

We will consider matrices of the form

$$
\mathcal{A}=\left(\begin{array}{cccc}
\alpha_{11} & \alpha_{12} & \cdots & \alpha_{1 n} \\
\vdots & \vdots & \ddots & \vdots \\
\alpha_{m 1} & \alpha_{m 2} & \cdots & \alpha_{m n}
\end{array}\right),
$$

where $m, n \in \mathbb{N}$ and $\alpha_{i j} \in \mathbb{E}$ for $1 \leq i \leq m, 1 \leq j \leq n$; the natural numbers $m, n$ are always supposed to be standard. We use the common notation $\mathcal{A}=\left(\alpha_{i j}\right)_{m \times n}$. The transpose of the matrix $\mathcal{A}$ is defined by $\mathcal{A}^{T}=\left(v_{i j}\right)_{n \times m}$ with $v_{i j}=\alpha_{j i}$ for $1 \leq i \leq n, 1 \leq j \leq m$.

Definition 3.1. Let $m, n \in \mathbb{N}$. As usual, we denote the zero-matrix by $O$, and if $m=n$, we denote the $m \times m$ identity matrix by $I_{m}$. A matrix $\mathcal{O}=\left(\alpha_{i j}\right)_{m \times n}$ is called neutricial if all elements of $\mathcal{O}$ are neutrices, and zeroless if all of its entries are zeroless. If $\alpha_{i j}=a_{i j}+A_{i j}$ for all $1 \leq i \leq m, 1 \leq j \leq n$, the matrix $\left(a_{i j}\right)_{m \times n}$ is called a matrix of representatives and $N(\mathcal{A}):=\left(A_{i j}\right)_{m \times n}$ the associated neutricial matrix. We denote by $\mathcal{M}_{m, n}(\mathbb{E})$ the class of all $m \times n$ matrices over $\mathbb{E}$. When $m=n$ we simply write $\mathcal{M}_{n}(\mathbb{E})$. For $\mathcal{A}, \mathcal{B} \in \mathcal{M}_{m \times n}(\mathbb{E})$ we write $\mathcal{A} \subseteq \mathcal{B}$ if $\alpha_{i j} \subseteq \beta_{i j}$ for all $1 \leq i \leq m, 1 \leq j \leq n$.

Definition 3.2. For matrices $\mathcal{A}=\left(\alpha_{i j}\right)_{m \times n} \equiv\left(a_{i j}+A_{i j}\right)_{m \times n} \in \mathcal{M}_{m, n}(\mathbb{E})$ we define

$$
\bar{A}=\max _{\substack{1 \leq i \leq m \\ 1 \leq j \leq n}} A_{i j}, \underline{A}=\min _{\substack{1 \leq i \leq m \\ 1 \leq j \leq n}} A_{i j},|\bar{\alpha}|=\max _{\substack{1 \leq i \leq m \\ 1 \leq j \leq n}}\left|\alpha_{i j}\right|,|\underline{\alpha}|=\min _{\substack{1 \leq i \leq m \\ 1 \leq j \leq n}}\left|\alpha_{i j}\right| .
$$

Operations on $\mathcal{M}_{m, n}(\mathbb{E})$ are defined similarly as in classical linear algebra.

Definition 3.3. Let $m, n, p \in \mathbb{N}$. Let $\mathcal{A}=\left(\alpha_{i j}\right)_{m \times n} \in \mathcal{M}_{m, n}(\mathbb{E}), \mathcal{B}=\left(\beta_{i j}\right)_{m \times n} \in \mathcal{M}_{m, n}(\mathbb{E}), \mathcal{C}=\left(\gamma_{i j}\right)_{n \times p} \in \mathcal{M}_{n, p}(\mathbb{E})$ and $\lambda \in \mathbb{E}$. Then

$$
\begin{gathered}
\mathcal{A}+\mathcal{B}=\left(\alpha_{i j}+\beta_{i j}\right)_{m \times n} \\
\lambda \mathcal{A}=\left(\lambda \alpha_{i j}\right)_{m \times n} \\
\mathcal{A C}=\left(\mu_{i j}\right)_{m \times p}
\end{gathered}
$$

with $\mu_{i j}=\sum_{k=1}^{n} \alpha_{i k} \gamma_{k j}$ for $1 \leq i \leq m, 1 \leq j \leq p$. 
The additive structure of $\mathcal{M}_{m, n}(\mathbb{E})$ reflects the additive structure of $\mathbb{E}$, which is a commutative regular semigroup, and also a monoid, meaning that every element $\alpha=a+A$ has the individual neutral element $A=\alpha-\alpha$ [10], but there exists also a universal neutral element in the form of 0 .

Proposition 3.4. The structure $\mathcal{M}_{m, n}(\mathbb{E})$ is a commutative regular semigroup for addition. In fact, let $\mathcal{A}, \mathcal{B}, \mathcal{C} \in$ $\mathcal{M}_{m, n}(\mathbb{E})$. Then

1. $\mathcal{A}+(\mathcal{B}+\mathcal{C})=(\mathcal{A}+\mathcal{B})+C$.

2. $\mathcal{A}+\mathcal{B}=\mathcal{B}+\mathcal{A}$.

3. $\mathcal{A}+\mathcal{O}=\mathcal{A}$ if and only if $\mathcal{O}_{i j} \subseteq(N(\mathcal{A}))_{i j}$ for $1 \leq i \leq m, 1 \leq j \leq n$.

4. $\mathcal{A}+(-\mathcal{A})=N(\mathcal{A})$.

The structure $\mathcal{M}_{m, n}(\mathbb{E})$ is also a monoid, with neutral element $O$.

Proof. The associative law and commutative law for addition hold for external numbers, hence also for matrices. This makes $\mathcal{M}_{m, n}(\mathbb{E})$ a commutative semigroup for addition. As for Parts 3 and 4, let $\mathcal{A} \in \mathcal{M}_{m, n}(\mathbb{E})$. Then $\mathcal{A}+N(\mathcal{A})=\mathcal{A}$, and $\mathcal{A}+(-\mathcal{A})=N(\mathcal{A})$. Hence $\mathcal{A}+(-\mathcal{A}+\mathcal{A})=\mathcal{A}$, so the commutative semigroup $\mathcal{M}_{m, n}(\mathbb{E})$ is regular. If also $\mathcal{O} \in \mathcal{M}_{m, n}(\mathbb{E})$ is neutricial and $\mathcal{A}+\mathcal{O}=A$, then $N(\mathcal{A})+\mathcal{O}=N(\mathcal{A})$.

Clearly $\mathcal{A}+O=O+\mathcal{A}=\mathcal{A}$ for all $\mathcal{A} \in \mathcal{M}_{m, n}(\mathbb{E})$. Hence the matrix $O$ acts as a neutral element, which makes $\mathcal{M}_{m, n}(\mathbb{E})$ a monoid.

Because in Part 3 of Proposition 3.4 it holds that $\mathcal{O}_{i j} \subseteq N(\mathcal{A})_{i j}$ for all $1 \leq i \leq m, 1 \leq j \leq n$, the matrix $N(\mathcal{A})$ is in a sense a maximal individualized neutral element. If $\mathcal{A}$ has an element $\alpha$ which is not real, then $N(\mathcal{A})$ has a non-zero element. So, except for matrices with real elements we do not have $\mathcal{A}+(-\mathcal{A})=O$.

In the remaining part of this section we study multiplication and its interaction with addition. We will see that most of the usual properties hold for non-negative matrices and non-negative scalars, and outside these classes they still hold under quite general conditions.

For any external number $\alpha$ one has $0 . \alpha=0$ and $1 . \alpha=\alpha$; also the multiplication of external numbers is associative. With these properties, the proofs of the next propositions are straightforward.

Proposition 3.5. Let $\mathcal{A} \in \mathcal{M}_{m, n}(\mathbb{E})$. Then

1. $0 \mathcal{A}=O$.

2. $1 \mathcal{A}=\mathcal{A}$.

3. $\alpha(\beta \mathcal{A})=(\alpha \beta) \mathcal{A}$.

Proposition 3.6. Let $\mathcal{A} \in \mathcal{M}_{m, p}(\mathbb{E}), \mathcal{B} \in \mathcal{M}_{p, q}(\mathbb{E})$. Then

1. $I_{m} \mathcal{A}=\mathcal{A}=\mathcal{A} I_{p}$.

2. $(\mathcal{A B})^{T}=\mathcal{B}^{T} \mathcal{A}^{T}$.

It follows from the fact that the multiplication of external numbers is not distributive that scalar multiplication and the multiplication of matrices is not distributive over addition. Theorem 3.8 below presents conditions such that the distributive property does hold.

Definition 3.7. Let $\mathcal{A}=\left(\alpha_{i j}\right)_{m \times n}, \mathcal{B}=\left(\beta_{i j}\right)_{m \times n} \in \mathcal{M}_{m, n}(\mathbb{E})$. The matrices $\mathcal{A}$ and $\mathcal{B}$ are said to be not nearly opposite if $\alpha_{i j}$ and $\beta_{i j}$ are not nearly opposite for all $1 \leq i \leq m, 1 \leq j \leq n$.

Note that matrices with entries of the same sign, and in particular non-negative matrices are not nearly opposite.

Theorem 3.8. Let $\mathcal{A}=\left(\alpha_{i j}\right)_{m \times n} \in \mathcal{M}_{m, n}(\mathbb{E})$, and $\mathcal{B}=\left(\beta_{i j}\right)_{n \times p}, \mathcal{C}=\left(\gamma_{i j}\right)_{n \times p} \in \mathcal{M}_{n, p}(\mathbb{E})$. Let $\alpha, \beta \in \mathbb{E}$. 
1. If either $R(\alpha) \leq \min _{\substack{1 \leq i \leq m \\ 1 \leq j \leq n}} \max \left\{R\left(\beta_{i j}\right), R\left(\gamma_{i j}\right)\right\}$ or $\mathcal{B}$, C are not nearly opposite, then

$$
\alpha(\mathcal{B}+\mathcal{C})=\alpha \mathcal{B}+\alpha \mathcal{C}
$$

2. If either $\max _{\substack{1 \leq i \leq m \\ 1 \leq j \leq n}}\left\{R\left(\alpha_{i j}\right)\right\} \leq \max \{R(\alpha), R(\beta)\}$ or $\alpha, \beta$ are not nearly opposite, then $(\alpha+\beta) \mathcal{A}=\alpha \mathcal{A}+\beta \mathcal{A}$.

3. If either $\max _{\substack{1 \leq i \leq m \\ 1 \leq j \leq n}} R\left(\alpha_{i j}\right) \leq \min _{\substack{1 \leq i \leq m \\ 1 \leq j \leq n}} \max \left\{R\left(\beta_{i j}\right), R\left(\gamma_{i j}\right)\right\}$ or $\mathcal{B}$, C are not nearly opposite, then $\mathcal{A}(\mathcal{B}+\mathcal{C})=\mathcal{A B}+\mathcal{A C}$.

Proof. Part 1 and Part 2 follow directly from Theorem 2.10. As for Part 3, let $\mathcal{A}(\mathcal{B}+\mathcal{C})=\left(\mu_{i j}\right)_{m \times n}, \mathcal{A B}=\left(\lambda_{i j}\right)_{m \times p}$ and $\mathcal{A C}=\left(v_{i j}\right)_{m \times p}$. It follows from Theorem 2.10 in case $\max _{\substack{1 \leq i \leq m \\ 1 \leq j \leq n}} R\left(\alpha_{i j}\right) \leq \min _{\substack{1 \leq i \leq m \\ 1 \leq j \leq n}} \max \left\{R\left(\beta_{i j}\right), R\left(\gamma_{i j}\right)\right\}$, and from Proposition 2.14 in case $\mathcal{B}$, $\mathcal{C}$ are not nearly opposite, that $\alpha_{i j}\left(\beta_{r s}+\gamma_{r s}\right)=\alpha_{i j} \beta_{r s}+\alpha_{i j} \gamma_{r s}$ whenever $1 \leq i \leq$ $m, 1 \leq j, r \leq n, 1 \leq s \leq p$. As a result,

$$
\begin{aligned}
\mu_{i j} & =\alpha_{i 1}\left(\beta_{1 j}+\gamma_{1 j}\right)+\cdots+\alpha_{i n}\left(\beta_{n j}+\gamma_{n j}\right) \\
& =\left(\alpha_{i 1} \beta_{1 j}+\cdots+\alpha_{i n} \beta_{n j}\right)+\left(\alpha_{i 1} \gamma_{1 j}+\cdots+\alpha_{i n} \gamma_{n j}\right) \\
& =\lambda_{i j}+v_{i j} .
\end{aligned}
$$

The next corollary gives conditions for distributivity in the case of zeroless matrices, in terms of minimal or maximal relative uncertainty.

Corollary 3.9. Let $\mathcal{A}=\left(\alpha_{i j}\right)_{m \times n} \in \mathcal{M}_{m, n}(\mathbb{E}), \mathcal{B}=\left(\beta_{i j}\right)_{n \times p}, \mathcal{C}=\left(\gamma_{i j}\right)_{n \times p} \in \mathcal{M}_{n, p}(\mathbb{E})$ be zeroless matrices. Let $\alpha, \beta \in \mathbb{E}$.

1. If $R(\alpha) \leq \max \{\underline{B} / \bar{\beta}, \underline{C} / \bar{\gamma})\}$, then $\alpha(\mathcal{B}+\mathcal{C})=\alpha \mathcal{B}+\alpha \mathcal{C}$.

2. If $\bar{A} / \underline{\alpha} \leq \max \{R(\alpha), R(\beta)\}$, then $(\alpha+\beta) \mathcal{A}=\alpha \mathcal{A}+\beta \mathcal{A}$.

3. If $\bar{A} / \underline{\alpha} \leq \max \{\underline{B} / \bar{\beta}, \underline{C} / \bar{\gamma})\}$, then $\mathcal{A}(\mathcal{B}+\mathcal{C})=\mathcal{A B}+\mathcal{A C}$.

Proof. 1. For all $1 \leq i \leq n, 1 \leq j \leq p$ it holds that

$$
\max \{\underline{B} / \bar{\beta}, \underline{C} / \bar{\gamma})\} \leq \max \left\{R\left(\beta_{i j}\right), R\left(\gamma_{i j}\right)\right\} .
$$

Then the result follows from Part 1 of Theorem 3.8.

2. The result follows from the fact that $\max _{1 \leq i \leq m} R\left(\alpha_{i j}\right) \leq \bar{A} / \underline{\alpha}$ and from Part 2 of Theorem 3.8.

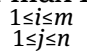

3. As in the proof of Part 1, formula (3) holds for all $1 \leq i \leq n, 1 \leq j \leq p$. Then the distributivity property is a consequence of Part 2 and Part 3 of Theorem 3.8 .

The subdistributivity property for external numbers implies the following general properties of subdistributivity for scalar multiplication and multiplication of matrices. The proofs are immediate.

Proposition 3.10. Let $\mathcal{A}=\left(\alpha_{i j}\right)_{m \times n}, \mathcal{B}=\left(\beta_{i j}\right)_{m \times n} \in \mathcal{M}_{m, n}(\mathbb{E})$, $\mathcal{C}=\left(\gamma_{i j}\right)_{n \times p}, \mathcal{D}=\left(v_{i j}\right)_{n \times p} \in \mathcal{M}_{n, p}(\mathbb{E})_{\text {. Let }}$ $\alpha, \beta \in \mathbb{E}$. Then

1. $\alpha(\mathcal{A}+\mathcal{B}) \subseteq \alpha \mathcal{A}+\alpha \mathcal{B}$.

2. $(\alpha+\beta) \mathcal{A} \subseteq \alpha \mathcal{A}+\beta \mathcal{A}$.

3. $\mathcal{A}(\mathcal{C}+\mathcal{D}) \subseteq \mathcal{A C}+\mathcal{A D}$.

4. $(\mathcal{A}+\mathcal{B}) \mathcal{C} \subseteq \mathcal{A} \mathcal{C}+\mathcal{B} \mathcal{C}$.

The fact that the distributivity law is not valid in general implies that the multiplication of matrices is not associative. The following example is taken from [22, p.35]. 
Example 3.11. Let $\mathcal{A}=\left(\begin{array}{ll}1 & 1 \\ 0 & 0\end{array}\right), \mathcal{B}=\left(\begin{array}{cc}1 & 0 \\ -1 & 0\end{array}\right), \mathcal{C}=\left(\begin{array}{l}\oslash \\ \oslash\end{array}\right)$. One has

$$
(\mathcal{A B}) \mathcal{C}=\left(\left(\begin{array}{ll}
1 & 1 \\
0 & 0
\end{array}\right)\left(\begin{array}{cc}
1 & 0 \\
-1 & 0
\end{array}\right)\right)\left(\begin{array}{l}
\oslash \\
\oslash
\end{array}\right)=\left(\begin{array}{l}
0 \\
0
\end{array}\right)
$$

and

$$
\mathcal{A}(\mathcal{B C})=\left(\begin{array}{ll}
1 & 1 \\
0 & 0
\end{array}\right)\left(\left(\begin{array}{cc}
1 & 0 \\
-1 & 0
\end{array}\right)\left(\begin{array}{l}
\oslash \\
\oslash
\end{array}\right)\right)=\left(\begin{array}{ll}
1 & 1 \\
0 & 0
\end{array}\right)\left(\begin{array}{l}
\oslash \\
\oslash
\end{array}\right)=\left(\begin{array}{l}
\oslash \\
0
\end{array}\right)
$$

So $(\mathcal{A B}) \mathcal{C} \neq \mathcal{A}(\mathcal{B C})$.

However, the subdistributivity of multiplication of external numbers, as shown in Corollary 2.7, implies the following properties of inclusion.

Proposition 3.12. Let $\mathcal{A}=\left(\alpha_{i j}\right)_{m \times n} \in \mathcal{M}_{m, n}(\mathbb{E}), \mathcal{B}=\left(\beta_{i j}\right)_{n \times p} \in \mathcal{M}_{n, p}(\mathbb{E})$ and $\mathcal{C}=\left(\gamma_{i j}\right)_{p \times q} \in \mathcal{M}_{p, q}(\mathbb{E})$. Then

1. $(\mathcal{A B}) \mathcal{C} \subseteq \mathcal{A}(\mathcal{B C})$ if $\mathcal{A}$ is a real matrix or $\mathcal{B}$, $\mathcal{C}$ are both non-negative.

2. $\mathcal{A}(\mathcal{B C}) \subseteq(\mathcal{A B}) \mathcal{C}$ if $\mathcal{C}$ is a real matrix or $\mathcal{A}, \mathcal{B}$ are both non-negative.

Proof. Let $\mathcal{A B} \equiv \mathcal{D} \equiv\left(\delta_{i j}\right)_{m \times p}, \mathcal{B C} \equiv \mathcal{E} \equiv\left(\varepsilon_{i j}\right)_{n \times q},(\mathcal{A B}) \mathcal{C} \equiv\left(\eta_{i j}\right)_{m \times q}$ and $\mathcal{A}(\mathcal{B C}) \equiv\left(\theta_{i j}\right)_{m \times q}$.

1. We have by subdistributivity for all $1 \leq i \leq m, 1 \leq k \leq q$

$$
\eta_{i k}=\sum_{j=1}^{p} \delta_{i j} \gamma_{j k}=\sum_{j=1}^{p}\left(\sum_{r=1}^{n} \alpha_{i r} \beta_{r j}\right) \gamma_{j k} \subseteq \sum_{j=1}^{p} \sum_{r=1}^{n} \alpha_{i r} \beta_{r j} \gamma_{j k} .
$$

If $\mathcal{A}$ is a real matrix, or else by non-negativity of $\mathcal{B} \mathcal{C}$, the last sum is equal to

$$
\sum_{r=1}^{n} \alpha_{i r}\left(\sum_{j=1}^{p} \beta_{r j} \gamma_{j k}\right)=\theta_{i k} .
$$

2. The proof is similar to the proof of Part 1.

We below provide conditions for the associative law for the multiplication of matrices to be valid.

Theorem 3.13. Let $\mathcal{A}=\left(\alpha_{i j}\right)_{m \times n} \in \mathcal{M}_{m, n}(\mathbb{E}), \mathcal{B}=\left(\beta_{i j}\right)_{n \times p} \in \mathcal{M}_{n, p}(\mathbb{E})$ and $\mathcal{C}=\left(\gamma_{i j}\right)_{p \times q} \in \mathcal{M}_{p, q}(\mathbb{E})$. Assume for every $i, j$ we have the left-hand side and right-hand side distributivity properties

$$
\begin{aligned}
\sum_{k=1}^{n} \alpha_{i k} \sum_{r=1}^{p} \beta_{k r} \gamma_{r j} & =\sum_{k=1}^{n} \sum_{r=1}^{p} \alpha_{i k}\left(\beta_{k r} \gamma_{r j}\right), \\
\sum_{r=1}^{p} \sum_{k=1}^{n}\left(\alpha_{i k} \beta_{k r}\right) \gamma_{r j} & =\sum_{r=1}^{p}\left(\sum_{k=1}^{n} \alpha_{i k} \beta_{k r}\right) \gamma_{r j} .
\end{aligned}
$$

Then $\mathcal{A}(\mathcal{B C})=(\mathcal{A B}) \mathcal{C}$.

Proof. By (4) we have $\mathcal{A}(\mathcal{B C})=\sum_{k=1}^{n} \sum_{r=1}^{p} \alpha_{i k}\left(\beta_{k r} \gamma_{r j}\right)$, and by (5) we have $(\mathcal{A B}) \mathcal{C}=\sum_{r=1}^{p} \sum_{k=1}^{n}\left(\alpha_{i k} \beta_{k r}\right) \gamma_{r j}$. Then the associativity property of the product of external numbers implies that $\mathcal{A}(\mathcal{B C})=(\mathcal{A B}) \mathcal{C}$.

Corollary 3.14. Let $\mathcal{A}=\left(\alpha_{i j}\right)_{m \times n} \in \mathcal{M}_{m, n}(\mathbb{E}), \mathcal{B}=\left(\beta_{i j}\right)_{n \times p} \in \mathcal{M}_{n, p}(\mathbb{E})$ and $\mathcal{C}=\left(\gamma_{i j}\right)_{p \times q} \in \mathcal{M}_{p, q}(\mathbb{E})$. Then $\mathcal{A}(\mathcal{B C})=(\mathcal{A B}) \mathcal{C}$ if either of the following conditions are satisfied: 
1. $\mathcal{A}$ and $\mathcal{C}$ are both real matrices.

2. $\mathcal{B}$ is a neutricial matrix.

3. $\mathcal{A}, \mathcal{B}, \mathcal{C}$ are all non-negative matrices.

Proof. In case $\mathcal{A}$ and $\mathcal{C}$ are both real matrices, the left-hand side and right-hand side distributivity properties (4) and (5) hold by Proposition 2.12, because real numbers are precise. In case $\mathcal{B}$ is a neutricial matrix they hold because $\mathcal{B C}$ and $\mathcal{A B}$ are neutricial. The properties (4) and (5) also hold if $\mathcal{A}, \mathcal{B}, \mathcal{C}$ are all non-negative matrices, because distributivity always holds when external numbers have the same sign. Then the result follows from Theorem 3.13.

Obviously, the above associative property continues to hold if the entries of each matrix all have the same sign.

The conditions of Corollary 3.14 are in a sense minimal conditions for associativity. They guarantee that the distributive properties of (4) and (5) hold term-by-term, but this is not necessary. To illustrate this, consider formula (4). For $1 \leq k \leq n$, put $s_{k}=\alpha_{i k} \sum_{r=1}^{p} \beta_{k r} \gamma_{r j}, t_{k}=\sum_{r=1}^{p} \alpha_{i k}\left(\beta_{k r} \gamma_{r j}\right), S_{k}=N\left(s_{k}\right)$ and $T_{k}=N\left(t_{k}\right)$. Assume that $1 \leq k_{1} \neq k_{2} \leq n$ and $S_{k_{1}} \subset T_{k_{1}} \subset S_{k_{2}}=T_{k_{2}}$, i.e. distributivity holds for a term of the sum with bigger neutrix than the neutrix of another term for which proper subdistributivity may holds. Then the equality of sums (4) still hold, and this is sufficient to be able to prove the corresponding associativity property.

An important class of matrices is given by the non-negative matrices. It follows from the above results that the class of non-negative matrices satisfies all axioms of a vector space, except for the existence of inverse elements for addition, such a space was called a semi-vector space in [15]. Also distributivity and associativity of multiplication are respected.

Definition 3.15. A class $U$ is called a semi-vector space over $\mathbb{E}^{+}$if for all $u, v, w \in U$ and $\lambda, \mu \in \mathbb{E}^{+}$

1. $u+v \in U$.

2. $u+(v+w)=(u+v)+w$.

3. $u+v=v+u$.

4. $0 \in U$ and $u+0=0$.

5. $\lambda u \in U$.

6. $\lambda(\mu u)=(\lambda \mu) u$.

7. $1 u=u$.

8. $\lambda(u+v)=\lambda u+\lambda v$.

9. $(\lambda+\mu) u=\lambda u+\mu u$.

Theorem 3.16. Let $\mathcal{M}_{m \times n}^{+}\left(\mathbb{E}^{+}\right)$be the class of non-negative matrices over $\mathbb{E}^{+}$. Then $\mathcal{M}_{m \times n}^{+}\left(\mathbb{E}^{+}\right)$is a semi-vector space over $\mathbb{E}^{+}$. Moreover, whenever the product of non-negative matrices over $\mathbb{E}^{+}$is well-defined, it is distributive and associative.

Proof. Part 1 and Part 5 of Definition 3.15 follow from the fact that the sum and the product of two nonnegative external numbers are non-negative. The properties 2 - 4 follow from Proposition 3.4, with $O$ the neutral element. The properties 6 and 7 follow from Proposition 3.5, and Theorem 3.8 implies the properties 8 and 9. Let $\mathcal{A}, \mathcal{B}, \mathcal{C} \in \mathcal{M}_{m \times n}^{+}\left(\mathbb{E}^{+}\right)$. Then it follows from Part 3 of Theorem 3.8 that $\mathcal{A}(\mathcal{B}+\mathcal{C})=\mathcal{A B}+\mathcal{A C}$ and $(\mathcal{B}+\mathcal{C}) \mathcal{A}=\mathcal{B} \mathcal{A}+\mathcal{C} \mathcal{A}$, and from Part 3 of Corollary 3.14 that $\mathcal{A}(\mathcal{B C})=(\mathcal{A B}) \mathcal{C}$.

\section{Determinants}

We define determinants of matrices with external numbers in the usual way through sums of signed products of entries. We show that this value does not always correspond to the set of determinants of representatives. 
Common techniques for calculation often use distributivity, so they need to be applied with care, for they may modify the neutrix part. We show that this is the case for the Laplace expansion. Using this expansion we derive a lower bound for minors, and also an upper bound is derived. Then we give conditions for the validity of the sum property for determinants. To calculate determinants in practice often the operations of Gauss elimination are applied, in order to obtain a triangular matrix; in this context this means a matrix with neutrices below or above the diagonal. This process searches for opposite terms, a context where the distributivity law is no longer valid. The use of Gauss elimination with real coefficients is helpful, but even then we need sometimes conditions on the order of magnitude of minors and neutrix parts. In the final part we give a condition implying that the determinant of a triangular matrix equals the product of the elements on the diagonal.

\subsection{Definition of the determinant}

Definition 4.1. [21] Let $\mathcal{A}=\left(\alpha_{i j}\right)_{n \times n} \in \mathcal{M}_{n}(\mathbb{E})$. The determinant of $\mathcal{A}$ is the external number defined by

$$
\operatorname{det}(\mathcal{A})=\sum_{\sigma \in S_{n}} \operatorname{sgn}(\sigma) \alpha_{1 \sigma(1)} \alpha_{2 \sigma(2)} \ldots \alpha_{n \sigma(n)},
$$

where $S_{n}$ is the set of all permutations of $\{1, \ldots, n\}$. We often denote the determinant of the matrix $\mathcal{A}$ by $\Delta$.

It is not true in general that the above definition of determinant corresponds to the set of values of determinants of representatives. We have equality in the case of $n=1$ and $n=2$, but for $n \geq 3$ we make repeated use of the same representatives in different products, and thus do not respect the Minkovski rules of Definition 2.2 properly.

Indeed, for $n=1$ and $\mathcal{A} \equiv(\alpha)$, with $\alpha \in \mathbb{E}$, one has $\operatorname{det}(\alpha)=\{a \mid a \in \alpha\}$. For $n=2$, let $\mathcal{A}=\left(\begin{array}{ll}\alpha_{11} & \alpha_{12} \\ \alpha_{21} & \alpha_{22}\end{array}\right)$. Then we have the equality

$$
\operatorname{det}(\mathcal{A})=\alpha_{11} \alpha_{22}-\alpha_{21} \alpha_{12}=\left\{a_{11} a_{22}-a_{21} a_{12} \mid a_{i j} \in \alpha_{i j}, 1 \leq i, j \leq 2\right\},
$$

for the determinant is a Minkowski sum of Minkowski products.

Now let $n=3$ and

$$
\mathcal{A}=\left(\begin{array}{lll}
\alpha_{11} & \alpha_{12} & \alpha_{13} \\
\alpha_{21} & \alpha_{22} & \alpha_{23} \\
\alpha_{31} & \alpha_{32} & \alpha_{33}
\end{array}\right)
$$

Then it may no longer hold that the sum of products (6) is equal to the sum of products of representatives. As for the latter, the Minkovski rules of Definition 2.2 are not applied properly, because, say, for the terms $\alpha_{11} \alpha_{22} \alpha_{33}$ and $-\alpha_{11} \alpha_{23} \alpha_{32}$ we choose repeatedly the same representative $a_{11}$ of $\alpha_{11}$, as a consequence we get only part of the value given by (6). In particular this means that the value obtained by the Rule of Sarrus does not need to correspond to the set of values given by the Rule of Sarrus applied to representatives. We give here an example.

Example 4.2. Let $\varepsilon \simeq 0, \varepsilon \neq 0$ and

$$
\mathcal{A}=\left(\begin{array}{ccc}
1+\oslash & 0 & 0 \\
0 & 1 & 1+\varepsilon \\
0 & 1 & 1
\end{array}\right)
$$

Then $\operatorname{det}(\mathcal{A})=\oslash$. Let $\varepsilon^{\prime} \simeq 0$ and $\widehat{\mathcal{A}_{\varepsilon^{\prime}}}$ be defined by

$$
\widehat{\mathcal{A}_{\varepsilon^{\prime}}}=\left(\begin{array}{ccc}
1+\varepsilon^{\prime} & 0 & 0 \\
0 & 1 & 1+\varepsilon \\
0 & 1 & 1
\end{array}\right)
$$

and let $S=\left\{\operatorname{det}\left(\widehat{\mathcal{A}_{\varepsilon^{\prime}}}\right) \mid \varepsilon^{\prime} \in \oslash\right\}$. Then $S=\left\{-\varepsilon-\varepsilon \varepsilon^{\prime} \mid \varepsilon^{\prime} \in \oslash\right\}=-(1+\oslash) \varepsilon \subset \oslash$. 
The following properties of determinants are obvious and proved using similar arguments as in classical algebra.

Proposition 4.3. Let $\mathcal{A} \in \mathcal{M}_{n}(\mathbb{E})$.

1. $\operatorname{det}(\mathcal{A})=\operatorname{det}\left(\mathcal{A}^{T}\right)$, where $\mathcal{A}^{T}$ is the transpose of the matrix $\mathcal{A}$.

2. Let $\mathcal{B}$ be a matrix obtained from $\mathcal{A}$ by interchanging two rows. Then $\operatorname{det}(\mathcal{B})=-\operatorname{det}(\mathcal{A})$. One has $\operatorname{det}(\mathcal{B})=$ $\operatorname{det}(\mathcal{A})$ if $\operatorname{det}(\mathcal{A})$ is a neutrix.

3. The determinant of a matrix which has a row of neutrices is a neutrix.

4. The determinant of a matrix which has two identical rows (columns) is a neutrix.

We use the following notation for minors.

Notation 4.4. For $1 \leq k<n$ we denote by $\Delta_{j_{1} \ldots j_{k}}^{i_{1} \ldots i_{k}}$ the $k \times k$ minor of $\mathcal{A}$ containing only the rows $\left\{i_{1} \ldots i_{k}\right\}$ and columns $\left\{j_{1} \ldots j_{k}\right\}$ of $\mathcal{A}$. We may use the standard notation $\Delta_{i, j}$ to denote the $(i, j)$-minor of $\mathcal{A}$ given by the determinant of $(n-1) \times(n-1)$ submatrix of $\mathcal{A}$ which results from removing the $i^{\text {th }}$ row and the $j^{\text {th }}$ column of $\mathcal{A}$.

\subsection{Laplace expansion}

Because of subdistributivity, the Laplace expansion of a determinant along a column or a row may not be equal to the determinant. For example, if we expand the determinant in Example 4.2 along the first column we obtain that

$$
(1+\oslash) \operatorname{det}\left(\begin{array}{cc}
1 & 1+\varepsilon \\
1 & 1
\end{array}\right)-\operatorname{Odet}\left(\begin{array}{ll}
0 & 0 \\
1 & 1
\end{array}\right)+\operatorname{Odet}\left(\begin{array}{cc}
0 & 0 \\
1 & 1+\varepsilon
\end{array}\right)=-(1+\oslash) \varepsilon \subset \oslash .
$$

So using products of representatives or the Laplace expansion possibly reduces the neutrix part, and even may turn a neutricial determinant into a zeroless value. We come back to this subject when we discuss singular and non-singular matrices in Section 5.

In general the Laplace expansion of a determinant along a column (row) is always included in the determinant.

Proposition 4.5. [22] Let $\mathcal{A}=\left(\alpha_{i j}\right)_{n \times n} \in \mathcal{M}_{n}(\mathbb{E})$ and $\Delta=\operatorname{det}(\mathcal{A})$. Then for $1 \leq j \leq n$,

$$
(-1)^{j+1} \alpha_{1 j} \Delta_{1, j}+\cdots+(-1)^{j+n} \alpha_{n j} \Delta_{n, j} \subseteq \Delta .
$$

Proof. It follows from Part 2 of Proposition 4.3 that it suffices to prove the proposition for $j=1$. Let $S_{n}$ be the set of all permutations of $\{1, \ldots, n\}$ and $\sigma \in S_{n}$. The Laplace expansion along the first column and the 
property of subdistributivity yield

$$
\begin{aligned}
& \alpha_{11} \Delta_{1,1}-\alpha_{21} \Delta_{2,1}+\cdots+\alpha_{n 1}(-1)^{1+n} \Delta_{n, 1} \\
= & \alpha_{11} \sum_{\substack{\sigma \in S_{n} \\
\sigma(1)=1}} \operatorname{sgn}(\sigma) \alpha_{\sigma(2) 2} \cdots \alpha_{\sigma(n) n}+\alpha_{21} \sum_{\substack{\sigma \in S_{n} \\
\sigma(1)=2}} \operatorname{sgn}(\sigma) \alpha_{\sigma(2) 2} \cdots \alpha_{\sigma(n) n} \\
+ & \cdots+\alpha_{n 1} \sum_{\substack{\sigma \in S_{n} \\
\sigma(1)=n}} \operatorname{sgn}(\sigma) \alpha_{\sigma(2) 2} \cdots \alpha_{\sigma(n) n} \\
\subseteq & \sum_{\substack{\sigma \in S_{n} \\
\sigma(1)=1}} \alpha_{11}\left(\operatorname{sgn}(\sigma) \alpha_{\sigma(2) 2} \cdots \alpha_{\sigma(n) n}\right)+\sum_{\substack{\sigma \in S_{n} \\
\sigma(1)=2}} \alpha_{21}\left(\operatorname{sgn}(\sigma) \alpha_{\sigma(2) 2} \cdots \alpha_{\sigma(n) n}\right) \\
+ & \cdots+\sum_{\substack{\sigma \in S_{n} \\
\sigma(1)=n}} \alpha_{n 1}\left(\operatorname{sgn}(\sigma) \alpha_{\sigma(2) 2} \cdots \alpha_{\sigma(n) n)}\right) \\
= & \sum_{\sigma \in S_{n}} \operatorname{sgn}(\sigma) \alpha_{\sigma(1) 1} \cdots \alpha_{\sigma(n-1)(n-1)} \alpha_{\sigma(n) n}=\operatorname{det}\left(\begin{array}{ccc}
\alpha_{11} & \cdots & \alpha_{1 n} \\
\vdots & \ddots & \vdots \\
\alpha_{n 1} & \cdots & \alpha_{n n}
\end{array}\right)=\Delta .
\end{aligned}
$$

Equality for the Laplace expansion holds, if we expand along a column (row) such that the relative uncertainty of all elements in this column are less than or equal to those of all the remaining elements.

Theorem 4.6. Let $\mathcal{A}=\left(\alpha_{i j}\right)_{n \times n} \in \mathcal{M}_{n}(\mathbb{E})$. If there exists $k \in\{1, \ldots, n\}$ such that

$$
\max _{1 \leq i \leq n} R\left(\alpha_{i k}\right) \leq \min _{\substack{j \neq k \\ 1 \leq i, j \leq n}} R\left(\alpha_{i j}\right)
$$

then

$$
(-1)^{k+1} \alpha_{1 k} \Delta_{1, k}+\cdots+(-1)^{k+n} \alpha_{n k} \Delta_{n, k}=\Delta
$$

Proof. We only prove the theorem for $k=1$, the other cases are similar. The Laplace expansion along column $k=1$ yields

$$
\begin{aligned}
& \alpha_{11} \Delta_{1,1}-\alpha_{21} \Delta_{2,1}+\cdots+\alpha_{n 1}(-1)^{1+n} \Delta_{n, 1} \\
= & \alpha_{11} \sum_{\substack{\sigma \in S_{n} \\
\sigma(1)=1}} \operatorname{sgn}(\sigma) \alpha_{\sigma(2) 2} \cdots \alpha_{\sigma(n) n}+\alpha_{21} \sum_{\substack{\sigma \in S_{n} \\
\sigma(1)=2}} \operatorname{sgn}(\sigma) \alpha_{\sigma(2) 2} \cdots \alpha_{\sigma(n) n}+\cdots \\
& +\alpha_{n 1} \sum_{\substack{\sigma \in S_{n} \\
\sigma(1)=n}} \operatorname{sgn}(\sigma) \alpha_{\sigma(2) 2} \cdots \alpha_{\sigma(n) n} .
\end{aligned}
$$

Put $\beta_{i 1}^{\sigma}=\operatorname{sgn}(\sigma) \alpha_{\sigma(2) 2} \cdots \alpha_{\sigma(n) n}$ with $\sigma \in S_{n}, \sigma(1)=i$. We will show that

$$
\alpha_{i 1} \sum_{\substack{\sigma \in S_{n} \\ \sigma(1)=i}} \operatorname{sgn}(\sigma) \alpha_{\sigma(2) 2} \cdots \alpha_{\sigma(n) n}=\sum_{\substack{\sigma \in S_{n} \\ \sigma(1)=i}} \operatorname{sgn}(\sigma) \alpha_{i 1} \alpha_{\sigma(2) 2} \cdots \alpha_{\sigma(n) n}
$$

for all $1 \leq i \leq n$. By Proposition 2.11 and assumption (9),

$$
\begin{aligned}
R\left(\alpha_{i 1}\right) & \leq \max _{1 \leq i \leq n} R\left(\alpha_{i 1}\right) \leq \min _{\substack{1 \leq r \leq n \\
2 \leq s \leq n}} R\left(\alpha_{r s}\right) \\
& \leq \min _{\substack{1 \leq r \leq n \\
2 \leq s \leq n \\
r \neq i}} R\left(\alpha_{r s}\right) \leq \max _{\substack{1 \leq r \leq n \\
2 \leq s \leq n \\
r \neq i}} R\left(\alpha_{r s}\right)=R\left(\beta_{i 1}^{\sigma}\right) .
\end{aligned}
$$


Then by Proposition 2.12

$$
\alpha_{i 1} \sum_{\substack{\sigma \in S_{n} \\ \sigma(1)=i}} \operatorname{sgn}(\sigma) \alpha_{\sigma(2) 2} \cdots \alpha_{\sigma(n) n}=\sum_{\substack{\sigma \in S_{n} \\ \sigma(1)=i}} \operatorname{sgn}(\sigma) \alpha_{i 1} \alpha_{\sigma(2) 2} \cdots \alpha_{\sigma(n) n}
$$

for all $1 \leq i \leq n$. From (10) we conclude that

$$
\begin{aligned}
& \alpha_{11} \Delta_{1,1}-\alpha_{21} \Delta_{2,1}+\cdots+\alpha_{n 1}(-1)^{1+n} \Delta_{n, 1} \\
= & \sum_{\substack{\sigma \in S_{n} \\
\sigma(1)=1}} \operatorname{sgn}(\sigma) \alpha_{11} \alpha_{\sigma(2) 2} \cdots \alpha_{\sigma(n) n}+\sum_{\substack{\sigma \in S_{n} \\
\sigma(1)=2}} \operatorname{sgn}(\sigma) \alpha_{21} \alpha_{\sigma(2) 2} \cdots \alpha_{\sigma(n) n} \\
& +\cdots+\sum_{\substack{\sigma \in S_{n} \\
\sigma(1)=n}} \operatorname{sgn}(\sigma) \alpha_{n 1} \alpha_{\sigma(2) 2} \cdots \alpha_{\sigma(n) n} \\
= & \sum_{\sigma \in S_{n}} \operatorname{sgn}(\sigma) \alpha_{\sigma(1) 1} \alpha_{\sigma(2) 2} \cdots \alpha_{\sigma(n) n}=\operatorname{det}(\mathcal{A}) .
\end{aligned}
$$

\subsection{Reduced matrices and minors}

We extend the notion of reduced matrix to matrices such that the maximal absolute value of an element is of the form of $1+A$, with $A \subseteq \oslash$ a small neutrix. We give lower bounds for minors, which are useful when studying Gauss elimination. Also upper bounds are given, as well for the associated neutrices.

Definition 4.7. A matrix $\mathcal{A}=\left(\alpha_{i j}\right)_{m \times n} \in \mathcal{M}_{m, n}(\mathbb{E})$, with $|\bar{\alpha}|=1+A$ and $A \subseteq \oslash$, is called a reduced matrix.

Reduced matrices have in each column (row) a minor of $(n-1)^{t h}$-order at least of the same order of magnitude as the determinant. The result has some relevance for Gauss elimination, for pivots may be expressed in terms of minors [18], so it is better to be able to choose them not too small.

Proposition 4.8. [22] Let $\mathcal{A}=\left(\alpha_{i j}\right)_{n \times n} \in \mathcal{M}_{n}(\mathbb{E})$ be a reduced square matrix of order $n$. Suppose that $\Delta$ is zeroless. Then for each $j \in\{1, \ldots, n\}$ there exists $i \in\{1, \ldots, n\}$ such that

$$
\left|\Delta_{i, j}\right|>\oslash \Delta .
$$

Proof. For simplicity we prove only the case $j=1$, the other cases are proved analogously. By Proposition 4.5 one has

$$
\alpha_{11} \Delta_{1,1}-\alpha_{21} \Delta_{2,1}+\cdots+\alpha_{n 1}(-1)^{n+1} \Delta_{n, 1} \subseteq \Delta .
$$

Suppose that $\Delta_{i, 1} \subseteq \oslash \Delta$ for $1 \leq i \leq n$. Because the matrix is reduced, it holds that $\left|\alpha_{i j}\right| \leq 1+\oslash$ for $1 \leq i, j \leq n$. So $\alpha_{i 1} \Delta_{i, 1} \subseteq(1+\oslash) \oslash \Delta=\oslash \Delta$ for $1 \leq i \leq n$. Consequently,

$$
\alpha_{11} \Delta_{1,1}-\alpha_{21} \Delta_{2,1}+\cdots+\alpha_{n 1}(-1)^{n+1} \Delta_{n, 1} \subseteq \oslash \Delta .
$$

Hence $\alpha_{11} \Delta_{1,1}-\alpha_{21} \Delta_{2,1}+\cdots+\alpha_{n 1}(-1)^{n+1} \Delta_{n, 1} \subseteq \Delta \cap \oslash \Delta$, which is a contradiction to (1), for $\Delta$ is zeroless.

The proposition below gives an upper bound for all minors of a reduced matrix, and also for the corresponding neutrix parts.

Proposition 4.9. Let $\mathcal{A}=\left(\alpha_{i j}\right)_{n \times n} \in \mathcal{M}_{n}(\mathbb{E})$ be a reduced matrix. Let $k \in\{1, \ldots, n\}$ and $1 \leq i_{1}<\cdots<i_{k} \leq$ $n, 1 \leq j_{1}<\cdots<j_{k} \leq n$. Then

1. $\Delta_{j_{1} \ldots j_{k}}^{i_{1} \ldots i_{k}} \subset €$. 
2. $N\left(\Delta_{j_{1} \ldots j_{k}}^{i_{1} \ldots i_{k}}\right) \subseteq \bar{A}$. In particular $N(\Delta) \subseteq \bar{A}$.

Proof. Let $I=\left\{i_{1}, \ldots, i_{k}\right\}, J=\left\{j_{1}, \ldots, j_{k}\right\}$. Let $S_{k}$ be the set of all bijections $\sigma: I \rightarrow J$.

1. Because $\mathcal{A}$ is a reduced matrix, it follows that $\left|\alpha_{i j}\right| \leq 1+\oslash$ for all $1 \leq i, j \leq n$. So

$$
\begin{aligned}
\left|\Delta_{j_{1} \ldots j_{k}}^{i_{1} \ldots i_{k}}\right| & =\left|\sum_{\sigma \in S_{k}} \operatorname{sgn}(\sigma) \alpha_{i_{1} \sigma\left(i_{1}\right)} \ldots \alpha_{i_{k} \sigma\left(i_{k}\right)}\right| \\
& \leq \sum_{\sigma \in S_{k}}\left|\alpha_{i_{1} \sigma\left(i_{1}\right)}\right| \ldots\left|\alpha_{i_{k} \sigma\left(i_{k}\right)}\right| \leq \sum_{\sigma \in S_{k}}(1+\oslash)^{k} \\
& =k !(1+\oslash) .
\end{aligned}
$$

Because $n \in \mathbb{N}$ is standard and $k \leq n$, it follows that $k ! \supsetneqq E$. Consequently, $k !(1+\oslash) \supsetneqq E$. Hence $\Delta_{j_{1} \ldots j_{k}}^{i_{1} \ldots i_{k}} \subset £$.

2. Because $\mathcal{A}$ is a reduced matrix, it follows that $\left|\alpha_{i j}\right| \leq 1+\bar{A}$ for all $1 \leq i, j \leq n$, while $\bar{A} \subseteq \oslash$. So

$$
\begin{aligned}
N\left(\Delta_{j_{1} \ldots j_{k}}^{i_{1} \ldots i_{k}}\right) & =N\left(\sum_{\sigma \in S_{k}} \operatorname{sgn}(\sigma) \alpha_{i_{1} \sigma\left(i_{1}\right)} \ldots \alpha_{i_{k} \sigma\left(i_{k}\right)}\right) \\
& =\sum_{\sigma \in S_{k}} N\left(\alpha_{i_{1} \sigma\left(i_{1}\right)} \cdots \alpha_{i_{k} \sigma\left(i_{k}\right)}\right) \subseteq \sum_{\sigma \in S_{k}} N\left((1+\bar{A})^{k}\right) \\
& =\sum_{\sigma \in S_{k}} \bar{A}=k ! \bar{A}=\bar{A} .
\end{aligned}
$$

When $k=n$ we obtain that $N(\Delta) \subseteq \bar{A}$.

\subsection{Addition property}

The addition property $\operatorname{det}(\mathcal{C})=\operatorname{det}(\mathcal{A})+\operatorname{det}(\mathcal{B})$ when $\mathcal{B}$ is equal to $\mathcal{A}$, except for one line, and $\mathcal{C}$ is obtained from $\mathcal{A}$ and $\mathcal{B}$ by summing with respect to this line does not hold in full generality as shown in Example 4.10 below.

Example 4.10. Let $\mathcal{A}=\left(\begin{array}{cc}1 & 1 \\ 1+\oslash & 1+\oslash\end{array}\right), \mathcal{B}=\left(\begin{array}{cc}-1 & -1 \\ 1+\oslash & 1+\oslash\end{array}\right)$ and $\mathcal{C}=\left(\begin{array}{cc}0 & 0 \\ 1+\oslash & 1+\oslash\end{array}\right)$. Then $\operatorname{det}(\mathcal{A})=$ $\operatorname{det}(\mathcal{B})=\oslash$, while $\operatorname{det}(\mathcal{C})=0 \neq \oslash=\operatorname{det}(\mathcal{A})+\operatorname{det}(\mathcal{B})$.

General conditions for this addition property to hold are stated in the next proposition.

Proposition 4.11. Let $k \in\{1, \ldots, n\}$. Let $\mathcal{B}=\left(\beta_{i j}\right)_{n \times n}, \mathcal{C}=\left(\gamma_{i j}\right)_{n \times n} \in \mathcal{M}_{n}(\mathbb{E})$ be matrices which possibly differ at row $k$, i.e. $\beta_{i j}=\gamma_{i j}$ for all $1 \leq i, j \leq n, i \neq k$ where all $\beta_{k j}, \gamma_{k j} \in \mathbb{E}$. Let $\mathcal{A}=\left(\alpha_{i j}\right)_{n \times n} \in \mathcal{M}_{n}(\mathbb{E})$ be defined by

$$
\alpha_{i j}= \begin{cases}\beta_{i j} & \text { if } i \neq k, 1 \leq j \leq n \\ \beta_{k j}+\gamma_{k j} & \text { if } i=k, 1 \leq j \leq n\end{cases}
$$

Then

$$
\operatorname{det} \mathcal{A} \subseteq \operatorname{det}(\mathcal{B})+\operatorname{det}(\mathcal{C})
$$

Moreover, if

$$
\max _{\substack{1 \leq i, j \leq n \\ i \neq k}} R\left(\alpha_{i j}\right) \leq \max \left\{\min _{1 \leq j \leq n} R\left(\beta_{k j}\right), \min _{1 \leq j \leq n} R\left(\gamma_{k j}\right)\right\},
$$

or $\beta_{k j}$ and $\gamma_{k j}$ are not nearly opposite for all $1 \leq j \leq n$, then (11) holds with equality instead of inclusion. 
Proof. By subdistributivity and the fact that $\alpha_{i j}=\beta_{i j}=\gamma_{i j}$, for $i \neq k$, we have

$$
\begin{aligned}
\operatorname{det}(\mathcal{A}) & =\sum_{\sigma \in S_{n}} \operatorname{sgn}(\sigma) \alpha_{1 \sigma(1)} \cdots \alpha_{n \sigma(n)} \\
& =\sum_{\sigma \in S_{n}} \operatorname{sgn}(\sigma) \alpha_{1 \sigma(1)} \cdots \alpha_{(k-1) \sigma(k-1)}\left(\beta_{k \sigma(k)}+\gamma_{k \sigma(k)}\right) \alpha_{(k+1) \sigma(k+1)} \cdots \alpha_{n \sigma(n)} \\
& \subseteq \sum_{\sigma \in S_{n}} \operatorname{sgn}(\sigma) \beta_{1 \sigma(1)} \cdots \beta_{(k-1) \sigma(k-1)} \beta_{k \sigma(k)} \beta_{(k+1) \sigma(k+1)} \cdots \beta_{n \sigma(n)} \\
& +\sum_{\sigma \in S_{n}} \operatorname{sgn}(\sigma) \gamma_{1 \sigma(1)} \cdots \gamma_{(k-1) \sigma(k-1)} \gamma_{k \sigma(k)} \gamma_{(k+1) \sigma(k+1)} \cdots \gamma_{n \sigma(n)} \\
& =\operatorname{det}(\mathcal{B})+\operatorname{det}(\mathcal{C}) .
\end{aligned}
$$

We now assume (12). For each $\sigma \in S_{n}$, let $\lambda_{\sigma}=\operatorname{sgn}(\sigma) \alpha_{1 \sigma(1)} \cdots \alpha_{(k-1) \sigma(k-1)} \alpha_{(k+1) \sigma(k+1)} \cdots \alpha_{n \sigma(n)}$. By Proposition 2.11 one has

$$
R\left(\lambda_{\sigma}\right)=\max _{\substack{1 \leq i \leq n \\ i \neq k}} R\left(\alpha_{i \sigma(i)}\right) .
$$

From (12) one derives that $R\left(\lambda_{\sigma}\right) \leq \max _{\substack{1 \leq i, j \leq n \\ i \neq k}} R\left(\alpha_{i j}\right) \leq \max \left\{R\left(\beta_{k j}\right), R\left(\gamma_{k j}\right)\right\}$ for all $1 \leq j \leq n$. By Theorem 2.10 we have

$$
\lambda_{\sigma}\left(\beta_{k j}+\gamma_{k j}\right)=\lambda_{\sigma} \beta_{k j}+\lambda_{\sigma} \gamma_{k j},
$$

for all $1 \leq j \leq n$.

If $\beta_{k j}$ and $\gamma_{k j}$ are not nearly opposite, we also have (13). This means that for all $\sigma \in S_{n}$,

$$
\begin{aligned}
& \operatorname{sgn}(\sigma) \alpha_{1 \sigma(1)} \cdots \alpha_{(k-1) \sigma(k-1)}\left(\beta_{k \sigma(k)}+\gamma_{k \sigma(k)}\right) \alpha_{(k+1) \sigma(k+1)} \cdots \alpha_{n \sigma(n)} \\
= & \operatorname{sgn}(\sigma) \alpha_{1 \sigma(1)} \cdots \alpha_{(k-1) \sigma(k-1)} \beta_{k \sigma(k)} \alpha_{(k+1) \sigma(k+1)} \cdots \alpha_{n \sigma(n)} \\
+ & \operatorname{sgn}(\sigma) \alpha_{1 \sigma(1)} \cdots \alpha_{(k-1) \sigma(k-1)} \gamma_{k \sigma(k)} \alpha_{(k+1) \sigma(k+1)} \cdots \alpha_{n \sigma(n)} .
\end{aligned}
$$

As a result,

$$
\begin{aligned}
& \operatorname{det}(\mathcal{A})=\sum_{\sigma \in S_{n}} \operatorname{sgn}(\sigma) \alpha_{1 \sigma(1)} \cdots \alpha_{n \sigma(n)} \\
& \quad=\sum_{\sigma \in S_{n}} \operatorname{sgn}(\sigma) \alpha_{1 \sigma(1)} \cdots \alpha_{(k-1) \sigma(k-1)}\left(\beta_{k \sigma(k)}+\gamma_{k \sigma(k)}\right) \alpha_{(k+1) \sigma(k+1)} \cdots \alpha_{n \sigma(n)} \\
& =\sum_{\sigma \in S_{n}} \operatorname{sgn}(\sigma) \beta_{1 \sigma(1)} \cdots \beta_{(k-1) \sigma(k-1)} \beta_{k \sigma(k)} \beta_{(k+1) \sigma(k+1)} \cdots \beta_{n \sigma(n)} \\
& \quad+\sum_{\sigma \in S_{n}} \operatorname{sgn}(\sigma) \gamma_{1 \sigma(1)} \cdots \gamma_{(k-1) \sigma(k-1)} \gamma_{k \sigma(k)} \gamma_{(k+1) \sigma(k+1)} \cdots \gamma_{n \sigma(n)} \\
& =\operatorname{det}(\mathcal{B})+\operatorname{det}(\mathcal{C}) .
\end{aligned}
$$

\subsection{On Gauss-elimination}

The operations of Gauss-elimination as regards to determinants of a matrix $\mathcal{A}$ can be effectuated for rows and columns, and because $\operatorname{det}(\mathcal{A})=\operatorname{det}\left(\mathcal{A}^{T}\right)$, without restriction of generality we may consider only operations of rows.

The effect of interchanging two rows of a matrix has been indicated in Proposition 4.3.2.

Because of subdistributivity, the operations of multiplying a row by an external number, and of adding a multiple of one row to another, may generate inclusions instead of equalities. In the first case we may avoid this by taking the external number to be sufficiently sharp, in particular by taking a real number. Even this 
may not be sufficient in the second case in the presence of too big neutrices or too big elements in the matrix. Bounds are given to guarantee equality.

We start with the operation of multiplying a row by a scalar.

Proposition 4.12. Let $\alpha$ be an external number and $\mathcal{A}=\left(\alpha_{i j}\right)_{n \times n} \in \mathcal{M}_{n}(\mathbb{E})$. Assume that $R(\alpha) \leq \min _{\substack{1 \leq i \leq n \\ 1 \leq j \leq n}} R\left(\alpha_{i j}\right)$. Let $k \in\{1, \ldots, n\}$ and $\mathcal{B}=\left(\beta_{i j}\right)_{n \times n}$ with

$$
\beta_{i j}= \begin{cases}\alpha_{i j} & \text { if } i \neq k \\ \alpha \alpha_{i j} & \text { if } i=k\end{cases}
$$

for all $1 \leq j \leq n$. Then $\operatorname{det}(\mathcal{B})=\alpha \operatorname{det}(\mathcal{A})$. The result holds in particular for $\alpha \in \mathbb{R}$.

Proof. One has

$$
\begin{aligned}
\operatorname{det}(\mathcal{B}) & =\sum_{\sigma \in S_{n}} \operatorname{sgn}(\sigma) \beta_{1 \sigma(1)} \cdots \beta_{n \sigma(n)} \\
& =\sum_{\sigma \in S_{n}} \operatorname{sgn}(\sigma) \alpha_{1 \sigma(1)} \cdots \alpha_{(i-1) \sigma(i-1)} \alpha \alpha_{i \sigma(i)} \alpha_{(i+1) \sigma(i+1)} \cdots \alpha_{n \sigma(n)} .
\end{aligned}
$$

Put $\lambda_{\sigma}=\alpha_{1 \sigma(1)} \cdots \alpha_{(i-1) \sigma(i-1)} \alpha_{i \sigma(i)} \alpha_{(i+1) \sigma(i+1)} \cdots \alpha_{n \sigma(n)}$. Then $R\left(\lambda_{\sigma}\right)=\max _{1 \leq i \leq n} R\left(\alpha_{i \sigma(i)}\right)$ by Proposition 2.11. By the assumption,

$$
R(\alpha) \leq \min _{1 \leq i, j \leq n} R\left(\alpha_{i j}\right) \leq \max _{1 \leq i \leq n} R\left(\alpha_{i \sigma(i)}\right)=R\left(\lambda_{\sigma}\right)
$$

for all $\sigma \in S_{n}$. Then by Proposition 2.12

$$
\operatorname{det}(\mathcal{B})=\alpha \sum_{\sigma \in S_{n}} \operatorname{sgn}(\sigma) \alpha_{1 \sigma(1)} \cdots \alpha_{n \sigma(n)}=\alpha \operatorname{det}(\mathcal{A}) .
$$

Formulas (14) and (15) hold in particular if $\alpha \in \mathbb{R}$.

We may have a strict inclusion if we multiply a row by an external number $\alpha$ with relative uncertainty bigger than some elements of $\mathcal{A}$. For instance, let $\alpha=\oslash$ and $\mathcal{A}=\left(\begin{array}{ll}1 & 1 \\ 1 & 1\end{array}\right)$, and let $\mathcal{B}$ be obtained by multiplying the first row of $\mathcal{A}$ by $\alpha$, i.e. $\mathcal{B}=\left(\begin{array}{ll}\oslash & \oslash \\ 1 & 1\end{array}\right)$. Then $0=\alpha \operatorname{det}(\mathcal{A}) \subset \operatorname{det}(\mathcal{B})=\oslash$.

The operation of adding a scalar multiple of one row of a matrix of real numbers may again lead to inclusions, for we may blow up neutrices. For example, let $\mathcal{A}=\left(\begin{array}{ll}1 & 1 \\ \oslash & 1\end{array}\right)$ and $\omega$ be an unlimited number. Let $\mathcal{B}$ be the matrix which is obtained from the matrix $\mathcal{A}$ by adding a multiple $\omega$ of the second row to the first one. Then $\mathcal{B}=\left(\begin{array}{cc}1+\omega \oslash & 1+\omega \\ \oslash & 1\end{array}\right)$. We see that $\operatorname{det}(\mathcal{A})=1+\oslash$ while $\operatorname{det}(\mathcal{B})=\omega \oslash$, so a zeroless determinant is transformed into a neutrix containing it.

We present a general property on how determinants behave under the addition of a multiple of a row to another row, and derive from it a condition of invariance.

Theorem 4.13. Let $\mathcal{A}=\left(\alpha_{i j}\right)_{n \times n} \in \mathcal{M}_{n}(\mathbb{E}), \lambda \in \mathbb{E}$ and $1 \leq p \neq k \leq n$. Let $\mathcal{A}^{\prime}=\left(\alpha_{i j}^{\prime}\right)_{n \times n} \in \mathcal{M}_{n}(\mathbb{E})$ be defined by

$$
\alpha_{i j}^{\prime}= \begin{cases}\alpha_{i j} & \text { if } i \neq k \\ \alpha_{k j}+\lambda \alpha_{p j} & \text { if } i=k\end{cases}
$$

for $1 \leq j \leq n$. Assume that $R(\lambda) \leq \min _{\substack{1 \leq i \leq n \\ 1 \leq j \leq n}} R\left(\alpha_{i j}\right)$ and $|\bar{\alpha}|=\max _{\substack{1 \leq i \leq n \\ 1 \leq j \leq n}}\left|\alpha_{i j}\right|$ is zeroless. Then

1. $\operatorname{det}\left(\mathcal{A}^{\prime}\right) \subseteq \operatorname{det}(\mathcal{A})+\lambda \bar{\alpha}^{n-1} \bar{A}$. 
2. If $\lambda \bar{\alpha}^{n-1} \bar{A} \subseteq N(\operatorname{det}(\mathcal{A}))$ and $\max _{\substack{1 \leq i, j \leq n \\ i \neq k}} R\left(\alpha_{i j}\right) \leq \min _{1 \leq j \leq n} R\left(\alpha_{k j}\right)$, then $\operatorname{det}\left(\mathcal{A}^{\prime}\right)=\operatorname{det}(\mathcal{A})$.

Proof. 1. Let $1 \leq k \neq p \leq n$. Let $\mathcal{B}$ be the matrix obtained from $\mathcal{A}$ by replacing the row $k$ with a copy of row $p$; then $\mathcal{B}$ takes the form

$$
\mathcal{B}=\left(\begin{array}{cccc}
\alpha_{11} & \alpha_{12} & \cdots & \alpha_{1 n} \\
\vdots & \vdots & \ddots & \vdots \\
\alpha_{p 1} & \alpha_{p 2} & \cdots & \alpha_{p n} \\
\vdots & \vdots & \ddots & \vdots \\
\alpha_{p 1} & \alpha_{p 2} & \cdots & \alpha_{p n} \\
\vdots & \vdots & \ddots & \vdots \\
\alpha_{n 1} & \alpha_{n 2} & \cdots & \alpha_{n n}
\end{array}\right)
$$

By Propositions 4.11 and 4.12 we have

$$
\operatorname{det}\left(\mathcal{A}^{\prime}\right) \subseteq \operatorname{det}(\mathcal{A})+\lambda \operatorname{det}(\mathcal{B})
$$

Now $\operatorname{det}(\mathcal{B})$ is a neutrix since $\mathcal{B}$ has two identical rows. Because $|\bar{\alpha}|$ is zeroless, we may choose a non-zero representative $a \in \alpha$ such that $\left|\bar{\alpha}_{i j} / a\right| \leq 1+\oslash$ for all $1 \leq i, j \leq n$. Let $\mathcal{R}$ be obtained from $\mathcal{B}$ by dividing every entry by $a$, then $\mathcal{R}$ is a reduced matrix. Note that $\operatorname{det}\left(\begin{array}{cc}\alpha_{p j} & \alpha_{p j^{\prime}} \\ \alpha_{p j} & \alpha_{p j^{\prime}}\end{array}\right) \subseteq a \bar{A}$ for $1 \leq j<j^{\prime} \leq n$. This implies that $\operatorname{det}(\mathcal{R}) \subseteq \bar{A} / a$. Also, by Proposition 4.12 and Proposition 2.9.1

$$
\operatorname{det}(\mathcal{B})=a^{n} \operatorname{det}(\mathcal{R}) \subseteq a^{n} \bar{A} / a=a^{n-1} \bar{A}=\bar{\alpha}^{n-1} \bar{A} .
$$

The last equality holds because $\bar{\alpha}$ is zeroless. From (16) and (17) we $\operatorname{derive}$ that $\operatorname{det}\left(\mathcal{A}^{\prime}\right) \subseteq \operatorname{det}(\mathcal{A})+\lambda \bar{\alpha}^{n-1} \bar{A}$.

2. By Propositions 4.11 and 4.12 the inclusion (16) becomes now an equality, i.e.

$$
\operatorname{det}\left(\mathcal{A}^{\prime}\right)=\operatorname{det}(\mathcal{A})+\lambda \operatorname{det}(\mathcal{B})
$$

In addition, if $\lambda \bar{\alpha}^{n-1} \bar{A} \subseteq N(\operatorname{det}(\mathcal{A}))$, by (17) it holds that $\lambda \operatorname{det}(\mathcal{B}) \subseteq N(\operatorname{det}(\mathcal{A}))$. Then we obtain from (18) that $\operatorname{det}\left(\mathcal{A}^{\prime}\right)=\operatorname{det}(\mathcal{A})$.

Observe that the first condition of Theorem 4.13 is automatically satisfied if $\lambda \in \mathbb{R}$. For reduced matrices the first condition in Part 2 of Theorem 4.13 is simplified into $\lambda \bar{A} \subseteq N(\operatorname{det}(\mathcal{A}))$.

\subsection{Determinants of triangular matrices}

Classically we use Gauss-elimination to transform a determinant into a determinant of a triangular matrix, and then the determinant is the product of the elements on the diagonal. In the context of external numbers the techniques of Subsection 4.5 generate neutrices instead of zeros, and by Theorem 4.13 the neutrix of the determinant may be modified. Proposition 4.15 shows that the determinant of a triangular matrix may not be equal to the product of the entries on the diagonal, and again it will be needed to add a neutrix.

Definition 4.14. Let $\mathcal{A}=\left(\alpha_{i j}\right)_{n \times n} \in \mathcal{M}_{n}(\mathbb{E})$. The matrix $\mathcal{A}$ is called upper triangular if $\alpha_{i j}$ is a neutrix for all $1 \leq j<i \leq n$. The matrix $\mathcal{A}$ is called lower triangular if $\alpha_{i j}$ is a neutrix for all $1 \leq i<j \leq n$. An upper triangular or lower triangular matrix is called a triangular matrix.

A triangular matrix with determinant equal to a neutrix is given by $\mathcal{A}=\left(\begin{array}{ll}1 & \oslash \\ \omega & 1\end{array}\right)$, where $\omega$ is an unlimited number. Then $1=1 \cdot 1 \neq \operatorname{det}(\mathcal{A})=\omega \oslash$. Next proposition gives an upper bound for such neutrices. 
Proposition 4.15. Let $\mathcal{A}=\left(\alpha_{i j}\right)_{n \times n}$ be a triangular matrix. Assume that $\bar{\alpha}$ is zeroless. If $\mathcal{A}$ is reduced,

$$
\operatorname{det}(\mathcal{A}) \subseteq \alpha_{11} \alpha_{22} \cdots \alpha_{n n}+\bar{A}
$$

In general

$$
\operatorname{det}(\mathcal{A}) \subseteq \alpha_{11} \alpha_{22} \cdots \alpha_{n n}+\bar{\alpha}^{n-1} \bar{A} \text {. }
$$

As a result $\operatorname{det}(\mathcal{A})=\alpha_{11} \alpha_{22} \cdots \alpha_{n n}$ if $\mathcal{A}$ is reduced and $\bar{A} \subseteq N\left(\alpha_{11} \alpha_{22} \cdots \alpha_{n n}\right)$, and in general if $\bar{\alpha}^{n-1} \bar{A} \subseteq$ $N\left(\alpha_{11} \alpha_{22} \cdots \alpha_{n n}\right)$.

Proof. Without loss of generality, we suppose that $\mathcal{A}$ is an upper triangular matrix. We have

$$
\begin{aligned}
\operatorname{det}(\mathcal{A}) & =\sum_{\sigma \in S_{n}} \operatorname{sgn}(\sigma) \alpha_{1 \sigma(1)} \cdots \alpha_{n \sigma(n)} \\
& =\alpha_{11} \cdots \alpha_{n n}+\sum_{\substack{\sigma \in S_{n} \\
\exists i \in\{1, \ldots n\}, \sigma(i) \neq i}} \operatorname{sgn}(\sigma) \alpha_{1 \sigma(1)} \cdots \alpha_{n \sigma(n)} .
\end{aligned}
$$

Assume first that $\mathcal{A}$ is a reduced matrix. For $\sigma \in S_{n}$ such that $i \neq \sigma(i)$ for some $i$ with $1 \leq i \leq n$, there exists $k \in\{1, \ldots, n\}$ such that $k>\sigma(k)$. Then $\alpha_{k \sigma(k)} \equiv A_{k \sigma(k)}$ is a neutrix. As a consequence $\alpha_{1 \sigma(1)} \cdots \alpha_{n \sigma(n)}$ is also a neutrix. Also $\left|\alpha_{i j}\right| \leq 1+\oslash$ for all $1 \leq i, j \leq n$, hence $\alpha_{1 \sigma(1)} \cdots \alpha_{n \sigma(n)} \subseteq A_{k \sigma(k)} \subseteq \bar{A}$. Because $|\bar{\alpha}|=1+\oslash$, we derive from (20) that $\operatorname{det}(\mathcal{A}) \subseteq \alpha_{11} \cdots \alpha_{n n}+\bar{A}$.

Second, assume that $\mathcal{A}$ is an arbitrary matrix such that $\bar{\alpha}$ is zeroless. Let $a \in \bar{\alpha}$ be non-zero and $\mathcal{A}^{\prime}=\left(\alpha_{i j}^{\prime}\right)$ with $\alpha_{i j}^{\prime}=\alpha_{i j} / a$ for $1 \leq i, j, \leq n$. Then $\mathcal{A}^{\prime}$ is a reduced upper triangular matrix. $\operatorname{Also} \operatorname{det}(\mathcal{A})=a^{n} \operatorname{det}\left(\mathcal{A}^{\prime}\right) \subseteq$ $a^{n}\left(\alpha_{11}^{\prime} \cdots \alpha_{n n}^{\prime}+\bar{A} / a\right)=\alpha_{11} \cdots \alpha_{n n}+a^{n-1} \bar{A}=\alpha_{11} \cdots \alpha_{n n}+\bar{\alpha}^{n-1} \bar{A}$. The remaining part of the theorem is now a direct consequence.

\section{Inverse matrices}

The additive inverse and multiplicative inverse of an external number $\alpha$ are defined up to a neutrix, for $\alpha-\alpha=$ $N(\alpha)$ and, if $\alpha$ is zeroless, one has $\alpha / \alpha=1+R(\alpha)$ with $R(\alpha) \subseteq \oslash$. Proposition 3.4 shows that the additive inverse of a matrix of external numbers exists up to a neutricial matrix. We define the multiplicative inverse of a matrix of external numbers also with respect to a neutrix, contained in $\oslash$. This neutrix is an upper bound for the precision that can be obtained and the (not unique) inverse is defined in terms of inclusion.

The relationship between invertible matrices and non-singular matrices (matrices with zeroless determinant) is investigated, as well as the possibility to determine inverses with the help of cofactors. Theorem 5.6 states that such inverses exist if the maximal absolute value of the elements of the matrix is zeroless, and the determinant is not too small.

Definition 5.1. Let $\mathcal{A}=\left(\alpha_{i j}\right)_{n \times n} \in \mathcal{M}_{n}(\mathbb{E})$. The matrix $\mathcal{A}$ is called non-singular if $\operatorname{det}(\mathcal{A})$ is zeroless. Otherwise we call it singular.

Definition 5.2. Let $\mathcal{A} \in \mathcal{M}_{n}(\mathbb{E})$ be a square matrix, $N \subseteq \oslash$ be a neutrix and $\mathcal{J}_{n}(N)=\left(\delta_{i j}\right) \in \mathcal{M}_{n}(E)$ with $\delta_{i j}=\left\{\begin{array}{ll}1+N & \text { if } i=j \\ N & \text { if } i \neq j\end{array}\right.$ for all $1 \leq i, j \leq n$. The matrix $\mathcal{A}$ is said to be invertible with respect to $N$ if there exists a square matrix $\mathcal{B}=\left(\beta_{i j}\right)_{n \times n}$ such that $\mathcal{A B} \subseteq \mathcal{J}_{n}(N)$ and $\mathcal{B} \mathcal{A} \subseteq \mathcal{J}_{n}(N)$. Then $\mathcal{B}$ is called an inverse matrix of $\mathcal{A}$ with respect to $N$ and with abuse of notation we may write $\mathcal{B}=\mathcal{A}_{N}^{-1}$.

If $\mathcal{A}$ is invertible with respect to $N \subseteq \oslash$, it is invertible with respect to every neutrix $M$ with $N \subseteq M \subseteq \oslash$. In case $\mathcal{A}$ is a real square matrix, the inverse matrix of $\mathcal{A}$ with respect to 0 becomes the classical one and we simply write $\mathcal{A}^{-1}$. 
Definition 5.3. Let $n \in \mathbb{N}$ and $\mathcal{A} \in \mathcal{M}_{n}(\mathbb{E})$. Let $\mathcal{C}_{i j}=(-1)^{i+j} \Delta_{i, j}$ for $1 \leq i, j \leq n$. We call $\mathcal{C}=\left(\mathcal{C}_{i j}\right)_{n \times n}$ the cofactor matrix of $\mathcal{A}$.

Even if $\mathcal{A}$ is a non-singular matrix, the matrix $\frac{1}{\operatorname{det}(\mathcal{A})} \mathrm{e}^{T}$ is not always an inverse matrix of $\mathcal{A}$ with respect to a neutrix. Indeed, let $\varepsilon>0$ be infinitesimal and $\mathcal{A}=\left(\begin{array}{ll}1 & \oslash \\ 0 & \varepsilon\end{array}\right)$. Then $\operatorname{det}(\mathcal{A})=\varepsilon$ is zeroless, so $\mathcal{A}$ is non-singular. We have $\mathcal{B}=\frac{1}{\operatorname{det}(\mathcal{A})} \mathcal{e}^{T}=\left(\begin{array}{cc}1 & \frac{\oslash}{\varepsilon} \\ 0 & \frac{1}{\varepsilon}\end{array}\right)$. This implies that $\mathcal{A B}=\left(\begin{array}{cc}1 & \frac{\oslash}{\varepsilon} \\ 0 & 1\end{array}\right) \nsubseteq \mathcal{J}_{2}(N)$ for any $N \subseteq \oslash$. Hence $\mathcal{B}$ is not an inverse matrix of $\mathcal{A}$.

Definition 5.4. Let $\mathcal{A}=\left(\alpha_{i j}\right)_{m \times n} \in \mathcal{M}_{m, n}(\mathbb{E})$. A matrix $P=\left(a_{i j}\right)_{m \times n}$, with $a_{i j} \in \alpha_{i j}$ for all $1 \leq i \leq m, 1 \leq j \leq n$, is called a representative matrix of the matrix $\mathcal{A}$.

Theorem 5.5. Let $\mathcal{A}=\left(\alpha_{i j}\right)_{2 \times 2} \in \mathcal{M}_{2}(\mathbb{E})$ be an invertible matrix with respect to a neutrix $N \subseteq \oslash$. Then $\mathcal{A}$ is non-singular.

Proof. Suppose that $\mathcal{A}$ is singular. Then $0 \in \operatorname{det}(\mathcal{A})$. By (7) there exists a representative matrix $P$ of $\mathcal{A}$ such that $\operatorname{det}(P)=0$. Let $\mathcal{B}$ be an inverse matrix of $\mathcal{A}$ with respect to some neutrix $N \subseteq \oslash$, and $Q$ be a representative matrix of $\mathcal{B}$. Then

$$
\operatorname{det}(P Q)=\operatorname{det}(P) \operatorname{det}(Q)=0 .
$$

On the other hand, one has $\mathcal{A B} \subseteq \mathcal{J}_{2}(N)$. Now $P Q$ is a representative matrix of $\mathcal{J}_{2}(N)$, so $\operatorname{det}(P Q) \neq 0$, contradicting (21). Hence $\mathcal{A}$ is non-singular.

The result above does not hold any more for $n>2$. This is related to the fact that the set of determinants of representatives of a given matrix $\mathcal{A}$ may be strictly contained in $\operatorname{det}(\mathcal{A})$. In fact, every matrix of representatives of a singular matrix may be non-singular. For example, consider the matrix $\mathcal{A}=\left(\begin{array}{ccc}1+\oslash & 0 & 0 \\ 0 & 1 & 1+\varepsilon \\ 0 & 1 & 1\end{array}\right)$ with $\varepsilon \simeq 0, \varepsilon \neq 0$ of Example 4.2. It is singular with determinant equal to $\oslash$, but it was shown that the set of determinants of matrices of representatives is equal to $(1+\oslash) \varepsilon$, so they are all non-singular. Also $\mathcal{A}$ is invertible with respect to $\oslash$. Indeed, we may take $\mathcal{A}_{\oslash}^{-1}=\left(\begin{array}{ccc}\frac{1}{\varepsilon+1} & 0 & 0 \\ 0 & -\frac{1}{\varepsilon} & \frac{1+\varepsilon}{\varepsilon} \\ 0 & \frac{1}{\varepsilon} & -\frac{1}{\varepsilon}\end{array}\right)$. Then $\mathcal{A}_{\oslash}^{-1} \cdot \mathcal{A}=\mathcal{A} \cdot \mathcal{A}_{\oslash}^{-1}=$ $\left(\begin{array}{ccc}1+\oslash & 0 & 0 \\ 0 & 1 & 0 \\ 0 & 0 & 1\end{array}\right) \subseteq I_{\oslash}$

Theorem 5.6 below gives conditions such that non-singular matrices are invertible. If the matrix $\mathcal{A}$ is reduced, a converse holds if $\operatorname{det}(\mathcal{A})$ is not so small as to be an absorber of $\bar{A}$. In general $\operatorname{det}(\mathcal{A})$ should not be an absorber of $\bar{\alpha}^{n} \bar{A}$, where $\bar{\alpha}$ is supposed zeroless.

Theorem 5.6. Let $\mathcal{A}=\left(\alpha_{i j}\right)_{n \times n} \in \mathcal{M}_{n}(\mathbb{E})$ be a non-singular matrix. Assume that

1. $\bar{\alpha}$ is zeroless.

2. $\operatorname{det}(\mathcal{A})$ is not an absorber of $\bar{\alpha}^{n} \bar{A}$.

Then $\mathcal{A}$ is invertible with respect to $\bar{A} / \bar{\alpha}$ and $\mathcal{B} \equiv \frac{1}{\operatorname{det}(\mathcal{A})} \mathrm{e}^{T}$ is an inverse matrix with respect to $\bar{A} / \bar{\alpha}$.

Proof. Note that $\bar{A} / \bar{\alpha} \subseteq \oslash$, because $\bar{\alpha}$ is zeroless. We first assume that $\mathcal{A}$ is a reduced, non-singular matrix. Let $\mathcal{A}=\left(\alpha_{i j}\right)_{n \times n}$ with $\alpha_{i j}=a_{i j}+A_{i j}$. Let $P=\left(a_{i j}\right)_{n \times n}, K=\left(A_{i j}\right)_{n \times n}$ and $\Delta=d+D$ with $d=\operatorname{det}(P) \neq 0$. Let $Q=\left(b_{i j}\right)_{n \times n}$ 
be the inverse matrix of $P$, then $b_{i j}=\frac{c_{i j}^{T}}{d}$, with $R=\left(c_{i j}\right)_{n \times n}$ the matrix of cofactors for $P$. Then the cofactor matrix is of the form $\mathcal{C}=\left(c_{i j}+C_{i j}\right)_{n \times n} \equiv\left(\gamma_{i j}\right)_{n \times n}$, and we define $M=\left(C_{i j}\right)_{n \times n}$ and $\mathcal{B}=\frac{1}{\operatorname{det}(\mathcal{A})} \mathrm{e}^{T}=\left(b_{i j}+B_{i j}\right)_{n \times n}$, where $B_{i j}=\frac{1}{d}\left(C_{i j}^{T}+\frac{\gamma_{i j}^{T} D}{d}\right)$ for $1 \leq i, j \leq n$. Let $L=\left(B_{i j}\right)_{n \times n}$ and let $I_{n}$ be the identity matrix of order $n$.

We show that $B_{i j} \subseteq \bar{A} \subseteq \oslash$ for all $1 \leq i, j \leq n$. Observe that $D \subseteq \bar{A}$ and $C_{i j} \subseteq \bar{A}$ for $1 \leq i, j \leq n$ by Proposition 4.9.2, and that Proposition 4.9.1 implies that $d$ is limited and $\gamma_{i j} \subseteq E$ for all $1 \leq i, j \leq n$. So $B_{i j} \subseteq \frac{1}{d}\left(\bar{A}+\frac{\bar{A}}{d}\right)=\frac{\bar{A}}{d}+\frac{\bar{A}}{d^{2}}$ for $1 \leq i, j \leq n$. Also $\operatorname{det}(\mathcal{A}) / \bar{\alpha}^{n}=\operatorname{det}(\mathcal{A})$ is not an absorber of $\bar{A}$, so neither is $d$, and therefore $d \bar{A}=\bar{A}=\frac{\bar{A}}{d}$. Hence $B_{i j} \subseteq \bar{A} \subseteq \oslash$ for $1 \leq i, j \leq n$.

Next, we prove that

$$
N(\mathcal{A B})=P L+K L+Q K \subseteq(\bar{A})_{n \times n} \subseteq(\oslash)_{n \times n} .
$$

Indeed, since $P \subseteq(E)_{n \times n}$ and $L \subseteq(\bar{A})_{n \times n}$, we derive that

$$
P L \subseteq(E)_{n \times n}(\bar{A})_{n \times n}=(\bar{A})_{n \times n} .
$$

Also $K \subseteq(\bar{A})_{n \times n}$, which implies that

$$
K L \subseteq(\bar{A})_{n \times n}(\bar{A})_{n \times n} \subseteq(\bar{A})_{n \times n} .
$$

In addition,

$$
K Q=K \frac{1}{d}\left(c_{i j}^{T}\right)_{n \times n} \subseteq \frac{1}{d}(\bar{A})_{n \times n}(E)_{n \times n}=\frac{1}{d}(\bar{A})_{n \times n}=(\bar{A})_{n \times n} .
$$

Then (22) follows from (23)-(25).

As a consequence $\mathcal{A B}=P Q+P L+K Q+K L \subseteq I_{n}+(\bar{A})_{n \times n}=\mathcal{J}_{n}(N)$. Similarly, we derive that $\mathcal{B} \mathcal{A} \subseteq \mathcal{J}_{n}(N)$. Hence $\mathcal{B}=\frac{1}{\operatorname{det}(\mathcal{A})} \mathrm{e}^{T}$ is an inverse matrix of $\mathcal{A}$ with respect to $A$.

We now assume that $\mathcal{A}=\left(\alpha_{i j}\right)_{n \times n} \in \mathcal{M}_{n}(\mathbb{E})$ is an arbitrary non-singular matrix such that $\bar{\alpha}$ is zeroless. Let $a \in \bar{\alpha}$, which is non-zero. Then $\mathcal{A}=a \mathcal{G}$ where $\mathcal{G}=\left(\alpha_{i j} / a\right)_{n \times n} \equiv\left(\eta_{i j}\right)$ is a reduced matrix. Because $\mathcal{A}$ is non-singular, the matrix $\mathcal{G}$ is non-singular. Also $\bar{\eta}=\bar{\alpha} / a$ is zeroless. Let $\eta_{i j}=g_{i j}+G_{i j}$ for all $1 \leq i, j \leq n$ and $\bar{G}=\max _{1 \leq i, j \leq n} G_{i j}=\frac{\bar{A}}{a} \subseteq \oslash$. Also $\operatorname{det}(\mathcal{A}) / \bar{\alpha}^{n}$ is not an absorber of $\bar{A}$, hence

$$
\bar{G}=\frac{\bar{A}}{a} \subseteq \frac{1}{a}\left(\frac{\operatorname{det}(\mathcal{A})}{\bar{\alpha}^{n}} \bar{A}\right)=\frac{1}{a}\left(\frac{\operatorname{det}(\mathcal{A})}{a^{n}} \bar{A}\right)=\operatorname{det}(\mathcal{G}) \frac{\bar{A}}{a}=\operatorname{det}(\mathcal{G}) \bar{G},
$$

implying that $\operatorname{det}(\mathcal{G})$ is not an absorber of $\bar{G}$. Since $\mathcal{G}$ is reduced, by the above argument $\mathcal{G}^{-1}=\frac{1}{\operatorname{det}(\mathcal{G})} \mathcal{D}^{T}$ is an inverse matrix of $\mathcal{G}$ with respect to $\bar{G} \subseteq \oslash$, where $\mathcal{D}$ is the cofactor matrix of $\mathcal{G}$. Let $\mathcal{H}=\frac{1}{a} \mathcal{G}^{-1}=\left(h_{i j}+H_{i j}\right)_{n \times n}$. Then $\mathcal{H}=\frac{1}{\operatorname{det} \mathcal{A}} \mathcal{e}^{T}$, with $\mathcal{C}$ the cofactor matrix of $\mathcal{A}$. Then $\mathcal{H}$ is an inverse matrix of $\mathcal{A}$ with respect to $\bar{G}$, because $\mathcal{A H}=a \mathcal{H} \frac{1}{a} \mathcal{G}^{-1}=\mathcal{G G}^{-1} \subseteq \mathcal{J}_{n}(\bar{G})$, and similarly, $\mathcal{H} \mathcal{A} \subseteq \mathcal{J}_{n}(\bar{G})$.

The choice of the representative matrix $P$ of $\mathcal{A}$ in the proof of Theorem 5.6 is arbitrary and $P^{-1}$ is always a representative of $\mathcal{A}^{-1}$. The final result of this section is an obvious consequence of the fact that $\left(P^{-1}\right)^{-1}=P$.

Corollary 5.7. Let $\mathcal{A}=\left(\alpha_{i j}\right)_{n \times n} \in \mathcal{M}_{n}(\mathbb{E})$ be invertible with respect to a neutrix $N \subseteq \oslash$ and let $\left(\mathcal{A}^{-1}\right)_{N}$ be an inverse matrix with respect to $N$ of $\mathcal{A}$. Then $\left(\mathcal{A}^{-1}\right)_{N}$ is invertible with respect to $N$ and $\mathcal{A}$ is an inverse matrix of $\mathcal{A}^{-1}$ with respect to $N$.

\section{Linear dependence and independence}

In this section we define the notions of linear dependence and linear independence for sets of vectors with external numbers. We give a characterization in terms of sets of vectors of representatives, and show that several common properties of linear independence continue to hold. 
Remark 6.1. In the present and next section it is always assumed that the number of components of a vector, and the cardinality of sets of vectors is standard finite.

We start by introducing some useful notions for external vectors.

Definition 6.2. Let $\beta=\left(\beta_{1}, \ldots, \beta_{n}\right) \in \mathbb{E}^{n}$. Then $\beta$ is called an external vector. A vector $b=\left(b_{1}, \ldots, b_{n}\right)$, where $b_{i} \in \beta_{i}$ for $1 \leq i \leq n$, is said to be a representative vector of $\beta$. Let $A_{1}, \ldots, A_{n}$ be neutrices. Then $A \equiv\left(A_{1}, \ldots, A_{n}\right)$ is called a neutrix vector.

Neutrix vectors can be seen as generalizations of the zero vector, and they are used in the following definition of linear dependence.

Definition 6.3. A set of vectors $V=\left\{\alpha_{1}, \ldots, \alpha_{m}\right\}$ where $\alpha_{i} \in \mathbb{E}^{n}$ for $1 \leq i \leq m$ is called linearly dependent if there exist real numbers $t_{1}, \ldots, t_{m} \in \mathbb{R}$, at least one of them being non-zero, and a neutrix vector $A$ such that

$$
t_{1} \alpha_{1}+\cdots+t_{m} \alpha_{m}=A .
$$

Otherwise, the set $V$ is called linearly independent.

In case $\left\{\alpha_{1}, \ldots, \alpha_{m}\right\} \subset \mathbb{R}^{n}$, this notion coincides with the conventional notion of linear algebra, for $A$, being a sum of real vectors, must be the zero vector. In the following characterization for linear independence $A$ also must be the zero vector.

Proposition 6.4. A set $V=\left\{\alpha_{1}, \cdots, \alpha_{m}\right\}$ of vectors in $\mathbb{E}^{n}$ is linearly independent if and only if the equality $t_{1} \alpha_{1}+\cdots+t_{m} \alpha_{m}=A$, where $A$ is a neutrix vector, implies $t_{1}=\cdots=t_{m}=0$ and $A$ is the zero vector.

Proof. Assume that $V$ is a linearly independent set of vectors and $t_{1} \alpha_{1}+\cdots+t_{m} \alpha_{m}=A$, where $A$ is a neutrix vector. If there exists $k \in\{1, \ldots, n\}$ such that $t_{k} \neq 0$, by Definition 6.3 the set $V$ is linearly dependent, a contradiction. Hence $t_{1}=\cdots=t_{n}=0$ and $A=(0, \ldots, 0)$.

Conversely, suppose that $V$ is a linearly dependent set. By Definition 6.3, there exist $t_{1}, \cdots t_{n} \in \mathbb{R}$ such that $t_{1} \alpha_{1}+\cdots+t_{m} \alpha_{m}=A$ where $A$ is a neutrix vector, while $t_{k} \neq 0$ for some $k$ with $1 \leq k \leq n$, a contradiction. Hence $V$ is linearly independent.

Example 6.5. Let $\varepsilon>0$ be infinitesimal. Then the vectors $\alpha_{1}=(1+\oslash, \varepsilon \oslash,-2+\varepsilon \notin), \alpha_{2}=(-2+\oslash, \varepsilon \varepsilon, 4+\varepsilon \notin)$ in $\mathbb{E}^{3}$ are linearly dependent, since $2 \alpha_{1}+\alpha_{2}=(\oslash, \varepsilon €, \varepsilon €)$ is a neutrix vector.

Example 6.6. The vectors $\alpha_{1}=(1+\oslash, \varepsilon \oslash), \alpha_{2}=(\oslash, 1+\varepsilon \varepsilon)$ with $\varepsilon>0, \varepsilon \simeq 0$ in $\mathbb{E}^{2}$ are linearly independent. Indeed, let $t_{1}, t_{2} \in \mathbb{R}$ and $A=\left(A_{1}, A_{2}\right)$ be a neutrix vector such that $t_{1} \alpha_{1}+t_{2} \alpha_{2}=A$. Then there exist vectors $x_{1}=(1+\eta, \varepsilon \zeta) \in \alpha_{1}$ and $x_{2}=(\vartheta, 1+\varepsilon \lambda) \in \alpha_{2}$, where $\eta, \zeta, \vartheta$ are infinitesimal and $\lambda$ is limited, such that $t_{1} x_{1}+t_{2} x_{2}=0$. It is equivalent with the system

$$
\left\{\begin{array}{l}
t_{1}(1+\eta)+t_{2} \vartheta=0 \\
t_{1} \zeta+t_{2}(1+\varepsilon \lambda)=0
\end{array}\right.
$$

Then $t_{1}=t_{2}=0$ because $\operatorname{det}\left(\begin{array}{cc}1+\eta & \vartheta \\ \zeta & 1+\varepsilon \lambda\end{array}\right) \neq 0$, so $t_{1} \alpha_{1}+t_{2} \alpha_{2}=0$. Hence the vectors $\alpha_{1}, \alpha_{2}$ are linearly independent.

The next proposition generalizes some common elementary properties of linear dependence and independence to external vectors. The proofs are obvious.

Proposition 6.7. Let $S=\left\{\xi_{1}, \cdots, \xi_{m}\right\}$ be a set of vectors in $\mathbb{E}^{n}$, and $k \in \mathbb{N}$ be standard.

1. If $S$ contains a neutrix vector it is linearly dependent. 
2. If the set $S$ is linearly dependent, any set of $k$ vectors including $S$ is linearly dependent.

3. If the set $S$ is linearly independent, any set of vectors included in $S$ is linearly independent.

In order to decide whether a set of vectors is linearly independent one often writes the set of vectors in matrix form, and then the usual tools are determinants and Gauss-elimination. We already saw that in order to be operational for matrices of external numbers, these tools should satisfy some conditions. So it is of interest to characterize linear independence and dependence of vectors in $\mathbb{E}^{n}$ via representatives, i.e. real numbers, and this is done in the theorem below.

Theorem 6.8. Let

$$
V=\left\{\xi_{1}=\left(\xi_{11}, \ldots, \xi_{1 n}\right), \xi_{2}=\left(\xi_{21}, \ldots, \xi_{2 n}\right), \ldots, \xi_{m}=\left(\xi_{m 1}, \ldots, \xi_{m n}\right)\right\} \subset \mathbb{E}^{n}
$$

be a set of vectors, with $\xi_{i j}=a_{i j}+A_{i j}$ for $1 \leq i \leq m$ and $1 \leq j \leq n$. Then

1. The set $V$ of vectors in $\mathbb{E}^{n}$ is linearly dependent if and only if whenever $1 \leq i \leq m$, there exist representative vectors $x_{i}=\left(x_{i 1}, \ldots, x_{i n}\right) \in \mathbb{R}^{n}$ of $\xi_{i}$ such that $\left\{x_{1}, \ldots, x_{m}\right\}$ is linearly dependent.

2. The set $V$ of vectors in $\mathbb{E}^{n}$ is linearly independent if and only if every set $\left\{x_{1}, \ldots, x_{m}\right\}$ of vectors in $\mathbb{R}^{n}$, where $x_{i} \in \xi_{i}$ for $1 \leq i \leq m$, is linearly independent.

Proof. 1. Suppose that the vectors $\xi_{1}, \ldots, \xi_{m}$ are linearly dependent. By Definition 6.3 there exist real numbers $t_{1}, \ldots, t_{m}$, at least one of them being non-zero, and a neutrix vector $A=\left(A_{1}, \ldots, A_{n}\right)$ such that

$$
t_{1} \xi_{1}+t_{2} \xi_{2}+\cdots+t_{m} \xi_{m}=A .
$$

Consequently $(0, \ldots, 0) \in t_{1} \xi_{1}+t_{2} \xi_{2}+\cdots+t_{m} \xi_{m}$. Hence there exist vectors $x_{i} \in \xi_{i}, i=1, \ldots, m$ such that $t_{1} x_{1}+t_{2} x_{2}+\cdots+t_{m} x_{m}=0$. That is, the set $\left\{x_{1}, \ldots, x_{m}\right\}$ is linearly dependent.

Conversely, suppose that there exists a linearly dependent set of vectors $V^{\prime}=\left\{x_{1}, \ldots, x_{m}\right\} \subset \mathbb{R}^{n}$, with $x_{i} \in \xi_{i}$ for $1 \leq i \leq m$; then let $x_{i}=\left(x_{i 1}, \ldots, x_{i n}\right)$ and $\xi_{i j}=x_{i j}+X_{i j}$, where $1 \leq j \leq n$. There exist real numbers $t_{1}, \ldots, t_{m}$, at least one of them being non-zero, such that $t_{1} x_{1}+t_{2} x_{2}+\cdots+t_{m} x_{m}=0$. Then $t_{1} x_{1 j}+\cdots+t_{m} x_{m j}=0$ for $1 \leq j \leq n$. So

$$
\begin{aligned}
t_{1} \xi_{1 j}+\cdots+t_{m} \xi_{m j} & =t_{1}\left(x_{1 j}+X_{1 j}\right)+\cdots+t_{m}\left(x_{m j}+X_{m j}\right) \\
& =t_{1} x_{1 j}+\cdots+t_{m} x_{m j}+t_{1} X_{1 j}+\cdots+t_{m} X_{m j} \\
& =t_{1} X_{1 j}+\cdots+t_{m} X_{m j} \equiv A_{j},
\end{aligned}
$$

where $A_{j}$ is a neutrix for $1 \leq j \leq n$. Hence $\left\{\xi_{1}, \ldots, \xi_{m}\right\}$ is linearly dependent.

2. The result follows from Part 1 by contraposition.

Observe that a set of linearly dependent vectors may have a set of linearly independent representative vectors.

Example 6.9. Let $\varepsilon>0$ be infinitesimal. Consider the set of vectors

$$
\left\{\xi_{1}=(\oslash, \oslash), \xi_{2}=(0, \varepsilon)\right\} .
$$

Then $\left\{\xi_{1}, \xi_{2}\right\}$ is linearly dependent by Proposition 6.7.1. Now we take $x_{1}=(\varepsilon, 0) \in \xi_{1}$ and $x_{2}=\xi_{2}$. Then $\left\{x_{1}, x_{2}\right\}$ is linearly independent.

We end with a generalization of a common property, which is a consequence of Theorem 6.8.

Proposition 6.10. Let $S=\left\{\xi_{1}, \cdots, \xi_{m}\right\}$ be a set of vectors in $\mathbb{E}^{n}$, where $m \in \mathbb{N}$ is standard. If $m>n$ the set $S$ is linearly dependent.

Proof. Suppose $S$ is linearly independent. For $1 \leq j \leq m$, let $x_{j}$ be a representative vector of $\xi_{j}$. By Theorem 6.8 the set $\left\{x_{1}, \cdots, x_{m}\right\}$ is linearly independent, a contradiction. Hence $S$ is linearly dependent. 


\section{Notions of rank}

Four notions of rank of a matrix are given. Three of them are obvious generalizations of classical properties, the row rank, which is the rank of the set of row vectors, the column rank with analogous definition and the minor rank, which is based on the maximal dimension of zeroless minors. However they may not be equal. For this reason the fourth notion of rank is introduced, called strict rank, based on both the minors and the rank of a representative matrix. The strict rank is not always defined, but if it exists, it is more operational than the other notions. For example, it permits to prove that the row rank and the minor rank are equal, and then they are also equal to the column rank, and in [36] it was helpful in solving singular systems of linear equations with coefficients and second member in terms of external numbers (the flexible systems of [21]).

Below we compare the ranks in various circumstances. The relation between determinants and determinants of representative matrices not being obvious for higher dimensions, some results are only derived for $1 \times 1$ and $2 \times 2$ matrices.

Definition 7.1. Let $\mathcal{A}=\left(\alpha_{i j}\right)$ be an $m \times n$ matrix over $\mathbb{E}$.

1. The row-rank of $\mathcal{A}$ is the maximal cardinality of a linearly independent set of row vectors of $\mathcal{A}$ and is denoted by $r(\mathcal{A})$, corresponding to the common notation of rank for sets of real vectors.

2. The column-rank of $\mathcal{A}$ is the maximal cardinality of a linearly independent set of column vectors of $\mathcal{A}$ and is denoted by $c(\mathcal{A})$.

3. The minor-rank of $\mathcal{A}$ is the largest natural number $m$ such that there exists a zeroless minor of order $m$ of $\mathcal{A}$. Then we write $\operatorname{mr}(\mathcal{A})=\mathrm{m}$.

4. The strict rank $\operatorname{sr}(\mathcal{A}) \equiv s$ of $\mathcal{A}$ is defined if both $\mathcal{A}$ has a zeroless minor of order $s$ and there exists a representative matrix $\hat{\mathcal{A}}$ of $\mathcal{A}$ with rank $s$.

Example 7.2. Let

$$
\mathcal{A}=\left(\begin{array}{ccc}
1+\oslash & 2+\oslash & -1+\varepsilon € \\
-2 & -4+\varepsilon & 2+\varepsilon \oslash
\end{array}\right)
$$

Then $\Delta_{12}^{12}=\Delta_{13}^{12}=\Delta_{23}^{12}=\oslash$, while $\Delta_{1}^{1}=1+\oslash$ is zeroless. Hence $\operatorname{mr}(\mathcal{A})=1$. It follows from the equality

$$
2(1+\oslash, 2+\oslash,-1+\varepsilon €)+(-2,-4+\varepsilon, 2+\varepsilon \oslash)=(\oslash, \oslash, \varepsilon €)
$$

that $r(\mathcal{A})=1$. The representative matrix

$$
\hat{\mathcal{A}}=\left(\begin{array}{ccc}
1 & 2 & -1 \\
-2 & -4 & 2
\end{array}\right)
$$

has rank 1 . Hence also $\operatorname{sr}(\mathcal{A})=1$.

Example 7.3. We reconsider the matrix

$$
\mathcal{A}=\left(\begin{array}{ccc}
1+\oslash & 0 & 0 \\
0 & 1 & 1+\varepsilon \\
0 & 1 & 1
\end{array}\right),
$$

where $\varepsilon \simeq 0, \varepsilon \neq 0$, of Example 4.2. Theorem 6.8.2 applied to the representative matrices (8) shows that the set of row vectors of $\mathcal{A}$ is linearly independent, so $r(\mathcal{A})=3$. Analogously $c(\mathcal{A})=3$. However we saw that $\operatorname{det}(\mathcal{A})=\oslash$, and $\left(\begin{array}{cc}1 & 1+\varepsilon \\ 1 & 1\end{array}\right)$ is a non-singular minor. $\operatorname{So} \operatorname{mr}(\mathcal{A})=2$, hence the minor rank is less than the row rank. Also the rank of the representative matrices (8), being equal to 3, is different from the minor rank. This means that the strict rank of $\mathcal{A}$ is not well-defined. 
In the remaining part of this section we investigate the relationship between the various ranks. In general the minor rank is less than or equal to the row rank (Theorem 7.5 ), and they are equal if the strict rank is welldefined (Theorem 7.6); then they are also equal to the column rank. We also have equality in some special cases of low rank (Propositions 7.9, 7.10 and 7.11) and if the matrix is non-singular, i.e. if the minor-rank is maximal (Theorem 7.4). We start with the latter theorem, since it is used further on.

Theorem 7.4. Let $\mathcal{A}=\left(\alpha_{i j}\right)_{n \times n} \in \mathcal{M}_{n}(\mathbb{E})$ be non-singular. Then $r(\mathcal{A})=n$.

Proof. Let $R=\left(a_{i j}\right)_{n \times n}$ be a representative matrix of $\mathcal{A}$. Because $\operatorname{det}(\mathcal{A})$ is zeroless, $\operatorname{det}(R) \neq 0$, so $R$ is nonsingular, with row rank equal to $n$. Hence the row vectors of $R$ are linearly independent. The choice of the $a_{i j}$ being arbitrary, by Theorem 6.8.2 also the row vectors of $\mathcal{A}$ are linearly independent. Hence $r(\mathcal{A})=n$.

Theorem 7.5. Assume $\mathcal{A}=\left(\alpha_{i j}\right)_{m \times n} \in \mathcal{M}_{m, n}(\mathbb{E})$ has minor rank $\operatorname{mr}(\mathcal{A})=\mathrm{r}$. Then there exists a linearly independent set of $r$ row vectors of $\mathcal{A}$. As a consequence $r(\mathcal{A}) \geq \operatorname{mr}(\mathcal{A})$.

Proof. Because $\operatorname{mr}(\mathcal{A})=\mathrm{r}$, we may suppose without loss of generality that the minor $\Delta_{1 \ldots r}^{1 \ldots .}$ is zeroless. Let $\xi_{i}=\left(\alpha_{i 1}, \ldots, \alpha_{i n}\right), 1 \leq i \leq m$ be row vectors of $\mathcal{A}$. Then $\xi_{i}^{\prime} \equiv\left(\alpha_{i 1}, \ldots, \alpha_{i r}\right)$ are vectors in $\mathbb{E}^{r}$ for $1 \leq i \leq m$. By Theorem 7.4 and the fact that $\Delta_{1 \ldots .}^{1} \ldots r$ is zeroless, the set of vectors $\left\{\xi_{1}^{\prime}, \ldots, \xi_{r}^{\prime}\right\}$ is linearly independent.

In order to prove that the set of vectors $\left\{\xi_{1}, \ldots, \xi_{r}\right\}$ is linearly independent, assume that $t_{1} \xi_{1}+\cdots+t_{r} \xi_{r}=$ $\left(A_{1}, \ldots, A_{n}\right)$, with $A_{1}, \ldots, A_{n}$ neutrices. Then $t_{1} \alpha_{1 j}+t_{2} \alpha_{2 j}+\cdots+t_{r} \alpha_{r j}=A_{j}$ for $1 \leq j \leq n$. It follows that $t_{1} \xi_{1}^{\prime}+\cdots+t_{r} \xi_{r}^{\prime}=\left(A_{1}, \ldots, A_{r}\right)$. Because $\left\{\xi_{1}^{\prime}, \ldots, \xi_{r}^{\prime}\right\}$ is linearly independent, it holds that $t_{1}=\cdots=t_{r}=0$. Hence the set of vectors $\left\{\xi_{1}, \ldots, \xi_{r}\right\}$ is linearly independent by Proposition 6.4.

Theorem 7.6. Let $\mathcal{A}$ be an $m \times n$ matrix over $\mathbb{E}$. If $\operatorname{sr}(\mathcal{A})=r$, then $\operatorname{mr}(\mathcal{A})=\mathrm{r}(\mathcal{A})=\mathrm{c}(\mathcal{A})=\mathrm{r}$.

Proof. First, because $\operatorname{sr}(\mathcal{A})=r$, there exists a zeroless minor of order $r$ of $\mathcal{A}$. By the definition of minor-rank $\operatorname{mr}(\mathcal{A}) \geq \mathrm{r}$. Let $k>r$ and $\mathcal{A}_{j_{1} \ldots j_{k}}^{i_{1} \ldots i_{k}}$ be a submatrix of order $k$ of $\mathcal{A}$. Because there exists a representative matrix $R \equiv\left(a_{i j}\right)_{m \times n}$ of $\mathcal{A}$ such that $\operatorname{rank}(R)=r$, we have $\operatorname{det}\left(R_{j_{1} \ldots j_{k}}^{i_{1} \ldots i_{k}}\right)=0$. So $\operatorname{det}\left(\mathcal{A}_{j_{1} \ldots j_{k}}^{i_{1} \ldots i_{k}}\right)$ is a neutrix. One concludes that $\operatorname{mr}(\mathcal{A})=\mathrm{r}$.

We now show that $r(\mathcal{A})=r$. Notice that $\operatorname{mr}(\mathcal{A})=\mathrm{r}$, by Theorem 7.5 there exists a linearly independent set of row vectors in $\mathcal{A}$ of cardinality at least $r$. On the other hand there exists a representative matrix $R^{\prime} \equiv$ $\left(a_{i j}^{\prime}\right)_{m \times n}$ of $\mathcal{A}$ with rank $r$. Without loss of generality, we may assume that $\operatorname{det}\left(R_{1 \ldots r}^{\prime 1 \ldots r}\right) \neq 0$. Let $i \in\{r+$ $1, \ldots, n\}$. Then the set of vectors

$$
\left\{a_{1}^{\prime}=\left(a_{11}^{\prime}, \ldots, a_{1 n}^{\prime}\right), \ldots, a_{r}^{\prime}=\left(a_{r 1}^{\prime}, \ldots, a_{r n}^{\prime}\right), a_{i}^{\prime}=\left(a_{i 1}^{\prime}, \ldots, a_{i n}^{\prime}\right)\right\}
$$

is linearly dependent. By Theorem 6.8.1 the set of vectors

$$
\left\{\alpha_{1}=\left(\alpha_{11}, \ldots, \alpha_{1 n}\right), \ldots, \alpha_{r}=\left(\alpha_{r 1}, \ldots, \alpha_{r n}\right), \alpha_{i}=\left(\alpha_{i 1}, \ldots, \alpha_{i n}\right)\right\}
$$

is linearly dependent. So the row rank of $\mathcal{A}$ is at most $r$. Combining, we obtain that $r(\mathcal{A})=r$.

One proves in analogous way that $c(\mathcal{A})=r$.

Theorem 7.7. Let $\mathcal{A}=\left(\alpha_{i j}\right)_{m \times n}$ be a matrix over $\mathbb{E}$. Assume that $r(\mathcal{A})=r$ and there is a zeroless minor of order $r$ of $\mathcal{A}$. Then $\operatorname{sr}(\mathcal{A})=r$ and $c(\mathcal{A})=r$.

Proof. A linearly independent set of row vectors of $\mathcal{A}$ has up to $r$ elements, so by Theorem 6.8 the same is true for every set of representative vectors $V=\left\{a_{1}, \ldots, a_{m}\right\}$, where $a_{i} \in \alpha_{i} \equiv\left(\alpha_{i 1}, \ldots, \alpha_{i n}\right)$ for $1 \leq i \leq m$. It follows that the rank of the representative matrix $R \equiv\left(a_{i j}\right)_{m \times n}$ is $r$. Because there exists a zeroless minor of order $r$ of $\mathcal{A}$, we have $\operatorname{sr}(\mathcal{A})=r$. Then $c(\mathcal{A})=r$ by Theorem 7.6.

Let $\mathcal{A}=\left(\alpha_{i j}\right)_{m \times n} \equiv\left(a_{i j}+A_{i j}\right)_{m \times n} \in \mathcal{M}_{m, n}(\mathbb{E})$. It was observed in Section 4 that only for $m=n \leq 2$ there is a straightforward relation between the determinants given by Definition 6 and determinants of representative matrices. This suggests that only for matrices of low rank there exists an obvious relation between the row 
rank and the minor rank, which can be established without recurring to the strict rank. Proposition 7.9 is a converse to Theorem 7.4 for $n=1$ and $n=2$ Proposition 7.10 considers the case that if the minor rank is equal to 1 , it is equal to the row rank, and then also equal to the strict rank, and Theorem 7.11 the converse case for ranks 1 or 2 . We start with some notation.

Notation 7.8. Let $\mathcal{A}=\left(\alpha_{i j}\right)_{m \times n} \equiv\left(a_{i j}+A_{i j}\right)_{m \times n} \in \mathcal{M}_{m, n}(\mathbb{E})$. For $1 \leq i \leq m$ we denote the $i^{\text {th }}$ row vector by $\alpha_{i} \equiv\left(\alpha_{i 1}, \cdots, \alpha_{i n}\right)$, and write $\bar{A}_{1} \equiv \max _{1 \leq i \leq m} A_{i 1}$ and $\underline{A}_{1}^{C}=\min _{\substack{2 \leq j \leq n \\ 1 \leq i \leq m}} A_{i j}$.

Proposition 7.9. Let $\mathcal{A}=\left(\alpha_{i j}\right)_{n \times n} \in \mathcal{M}_{n, n}(\mathbb{E})$. Assume that $r(\mathcal{A})=n$ and that $n=1$ or $n=2$. Then $\operatorname{mr}(\mathcal{A})=\mathrm{n}$.

Proof. The proposition is obvious if $n=1$. For $n=2$, by assumption $\left\{\alpha_{1}, \alpha_{2}\right\}$ is linearly independent. Let $a_{1}=\left(a_{11}, a_{12}\right)$ be a representative vector of $\alpha_{1}$, and $a_{2}=\left(a_{21}, a_{22}\right)$ be a representative vector of $\alpha_{2}$. By Theorem 6.8.2 the set $\left\{a_{1}, a_{2}\right\}$ is linearly independent. Hence $a_{11} a_{22}-a_{21} a_{12} \neq 0$. Because $a_{11}, a_{12}, a_{21}, a_{22}$ are arbitrary, it follows from $(7)$ that $\operatorname{det}(\mathcal{A})=\alpha_{11} \alpha_{22}-\alpha_{21} \alpha_{12}$ is zeroless. Hence $\operatorname{mr}(\mathcal{A})=2$.

Example 7.3 shows that Proposition 7.9 does no longer hold for $n \geq 3$; it also shows that the next proposition is not valid for $n \geq 2$.

Proposition 7.10. Let $\mathcal{A}=\left(\alpha_{i j}\right)_{m \times n} \in \mathcal{M}_{m, n}(\mathbb{E})$ be a reduced matrix. Assume that $\operatorname{mr}(\mathcal{A})=1$ and $\alpha_{11}$ is zeroless. Suppose that (i) $\frac{\bar{A}_{1}}{\alpha_{11}} \subseteq \underline{A}_{1}^{C}$ for $1 \leq i \leq m$, or (ii) all $A_{i j}$ are equal to some neutrix $A$, where $1 \leq i \leq m$ and $1 \leq j \leq n$. Then $r(\mathcal{A})=\operatorname{sr}(\mathcal{A})=1$.

Proof. We let $\alpha_{i j}=a_{i j}+A_{i j}$ for $1 \leq i \leq m$ and $1 \leq j \leq n$. The result is obvious for $m=1$. Assume that $1<m$. We will show that every set $\left\{\alpha_{1}, \alpha_{i}\right\}$ is linearly dependent, where $i \in\{2, \ldots, m\}$. In view of Theorem 7.7 we prove first that there exists a set of representative vectors

$$
\left\{a_{1}=\left(a_{11}, \ldots, a_{1 n}\right), a_{i}=\left(a_{i 1}, \ldots, a_{i n}\right)\right\}
$$

of $\left\{\alpha_{1}, \alpha_{i}\right\}$, such that the set of vectors $\left\{a_{1}, a_{i}\right\}$ is linearly dependent. To do so, we prove that there is a set of vectors

$$
\left\{a_{1}=\left(a_{11}, \ldots, a_{1 n}\right), a_{i}=\left(a_{i 1}, \ldots, a_{i n}\right)\right\},
$$

with $a_{p q} \in \alpha_{p q}, p \in\{1, i\}, q \in\{1, \ldots, n\}$ satisfying

$$
\operatorname{det}\left(\begin{array}{ll}
a_{11} & a_{1 j} \\
a_{i 1} & a_{i j}
\end{array}\right)=0,
$$

for $2 \leq j \leq n$.

For $j=2$, because $\operatorname{mr}(\mathcal{A})=1$, the determinant

$$
\operatorname{det}\left(\begin{array}{ll}
\alpha_{11} & \alpha_{12} \\
\alpha_{i 1} & \alpha_{i 2}
\end{array}\right)
$$

is a neutrix. Consequently, there exists $a_{p s} \in \alpha_{p s}$ for all $p \in\{1, i\}, s \in\{1,2\}$ such that

$$
\operatorname{det}\left(\begin{array}{ll}
a_{11} & a_{12} \\
a_{i 1} & a_{i 2}
\end{array}\right)=0 .
$$

Hence formula (27) is true for $j=2$. Let $k \in \mathbb{N}, 2<k \leq n$ be arbitrary. We need to prove that there is a column $a_{k}=\left(a_{1 k}, a_{i k}\right)^{T}$ such that $a_{p k} \in \alpha_{p k}$ for $p \in\{1, i\}$ and

$$
\operatorname{det}\left(\begin{array}{ll}
a_{11} & a_{1 k} \\
a_{i 1} & a_{i k}
\end{array}\right)=0,
$$


where $a_{11}, a_{i 1}$ are defined by (28). Again because $\operatorname{mr}(\mathcal{A})=1$, the determinant

$$
\operatorname{det}\left(\begin{array}{cc}
\alpha_{11} & \alpha_{1 k} \\
\alpha_{i 1} & \alpha_{i k}
\end{array}\right)
$$

is a neutrix. As a result, there exists a representative matrix $\left(\begin{array}{ll}a_{11}^{\prime} & a_{1 k}^{\prime} \\ a_{i 1}^{\prime} & a_{i k}^{\prime}\end{array}\right)$ with $a_{i j}^{\prime} \in \alpha_{i j}$ such that

$$
\operatorname{det}\left(\begin{array}{cc}
a_{11}^{\prime} & a_{1 k}^{\prime} \\
a_{i 1}^{\prime} & a_{i k}^{\prime}
\end{array}\right)=0
$$

As for case (i), we put

$$
d=a_{11}, t=\operatorname{det}\left(\begin{array}{cc}
a_{11} & a_{1 k}^{\prime} \\
a_{i 1} & a_{i k}^{\prime}
\end{array}\right)
$$

and

$$
\varepsilon_{11}=a_{11}-a_{11}^{\prime}, \varepsilon_{i 1}=a_{i 1}-a_{i 1}^{\prime}, \varepsilon_{i k}=-\frac{t}{d} .
$$

Observe first that $\varepsilon_{q 1} \in A_{q 1}$ for all $q \in\{1, i\}$. We show that also $\varepsilon_{i k} \in A_{i k}$. By (30) one has

$$
\begin{aligned}
t & =\operatorname{det}\left(\begin{array}{ll}
a_{11}^{\prime}+\varepsilon_{11} & a_{1 k}^{\prime} \\
a_{i 1}^{\prime}+\varepsilon_{i 1} & a_{i k}^{\prime}
\end{array}\right) \\
& =\operatorname{det}\left(\begin{array}{ll}
a_{11}^{\prime} & a_{1 k}^{\prime} \\
a_{i 1}^{\prime} & a_{i k}^{\prime}
\end{array}\right)+\operatorname{det}\left(\begin{array}{ll}
\varepsilon_{11} & a_{1 k}^{\prime} \\
\varepsilon_{i 1} & a_{i k}^{\prime}
\end{array}\right)=\varepsilon_{11} a_{i k}^{\prime}-\varepsilon_{i 1} a_{1 k}^{\prime} .
\end{aligned}
$$

Because $\varepsilon_{p 1} \in A_{p 1} \subseteq \bar{A}_{1}$ for $p \in\{1, i\}$ and $\left|a_{h k}^{\prime}\right| \leq\left|\alpha_{h k}\right| \leq 1+\oslash$ for $h \in\{1, i\}$, it holds that $t \in \bar{A}_{1}$. Also $d=a_{11} \in \alpha_{11}$. We conclude that

$$
\varepsilon_{i k}=-\frac{t}{d} \in \frac{\bar{A}_{1}}{d} \subseteq \underline{A}_{1}^{C} \subseteq A_{i k} .
$$

Hence $a_{p k} \in \alpha_{p k}$ for all $p \in\{1, i\}$ with $a_{. k}=\left(a_{1 k}, a_{i k}\right)^{T} \equiv\left(a_{1 k}^{\prime}, a_{i k}^{\prime}+\varepsilon_{i k}\right)^{T}$. In addition $a_{. k}$ satisfies formula (29), for

$$
\begin{aligned}
\operatorname{det}\left(\begin{array}{cc}
a_{11} & a_{1 k}^{\prime} \\
a_{i 1} & a_{i k}^{\prime}+\varepsilon_{i k}
\end{array}\right) & =t+\operatorname{det}\left(\begin{array}{cc}
a_{11} & 0 \\
a_{i 1} & \varepsilon_{i k}
\end{array}\right) \\
& =t+\varepsilon_{i k} d=t-\frac{t}{d} d=0 .
\end{aligned}
$$

As for case (ii), without loss of generality we assume that $\left|\alpha_{11}\right|$ is maximal. Put $u_{1}=\left(a_{11}, a_{i 1}\right)$. The set of column vectors

$$
\left\{u_{1}^{\prime}=\left(a_{11}^{\prime}, a_{i 1}^{\prime}\right)^{T}, u_{k}^{\prime}=\left(a_{1 k}^{\prime}, a_{i k}^{\prime}\right)^{T}\right\}
$$

is linearly dependent. As a consequence, there exist real numbers $s$ and $\delta_{11}, \delta_{i 1} \in A$ such that

$$
u_{k}^{\prime}=s u_{1}^{\prime}=s\left(u_{1}+\delta_{1}\right)=s u_{1}+s \delta_{1},
$$

where $\delta_{1} \equiv\left(\delta_{11}, \delta_{i 1}\right) \in(A, A)$ and $s=a_{1 k}^{\prime} / a_{11}^{\prime}$. Moreover $|s| \leq 1+\oslash$, since $\left|\alpha_{11}\right|$ is maximal. So $s \delta_{1} \in(A, A)$.

Put

$$
u_{k}=u_{k}^{\prime}-s \delta_{1} \equiv\left(a_{1 k}, a_{i k}\right)^{T} .
$$

Then $a_{q k} \in \alpha_{q k}$ for $q \in\{1, i\}$. By (31) one has $u_{k}=s u_{1}$, so $\left\{u_{1}, u_{k}\right\}$ is linearly dependent. Hence

$$
\operatorname{det}\left(\begin{array}{cc}
a_{11} & a_{1 k} \\
a_{i 1} & a_{i k}
\end{array}\right)=0 \text {, }
$$

which amounts again to (29).

In both cases, because $k$ is arbitrary, formula (27) holds for $2 \leq j \leq n$. We conclude that the set of vectors $\left\{a_{1}, a_{p}\right\}$ is linearly dependent. Then $\left\{\alpha_{1}, \alpha_{p}\right\}$ is linearly dependent for all $p \in\{2, \ldots, m\}$ by Theorem 6.8. So a linear independent set of row vectors of $\mathcal{A}$ cannot have more than one element, hence $r(\mathcal{A})=1$. The fact that $\operatorname{sr}(\mathcal{A})=1$ follows by Theorem 7.7. 
Proposition 7.11. Let $\mathcal{A}=\left(\alpha_{i j}\right)_{m \times n} \in \mathcal{M}_{m \times n}(\mathbb{E})$. Assume that $r(\mathcal{A})=r \leq \min \{m, n\}$. If (i) $r=1$ or (ii) $r=2$ and all $A_{i j}$ are equal to some neutrix $A$, then $\operatorname{mr}(\mathcal{A})=\mathrm{r}$. As a result, $\operatorname{sr}(\mathcal{A})=r$.

Proof. (i) Because the row rank of $\mathcal{A}$ is 1 , some $\alpha_{p q}$ is zeroless, where $p \in\{1, \ldots, m\}, q \in\{1, \ldots, n\}$. This implies that $\operatorname{mr}(\mathcal{A}) \geq 1$. Also $\operatorname{mr}(\mathcal{A}) \leq \mathrm{r}(\mathcal{A})=1$ by Theorem 7.5. It follows that $\operatorname{mr}(\mathcal{A})=1$.

(ii) By Theorem 7.5 it holds that $\operatorname{mr}(\mathcal{A}) \leq \mathrm{r}(\mathcal{A})=2$. Suppose that all minors of order 2 are neutricial. Then $\operatorname{mr}(\mathcal{A}) \leq 1$. If $\operatorname{mr}(\mathcal{A})=0$, then $r=0$, a contradiction. If $\operatorname{mr}(\mathcal{A})=1$, by part (ii) of Theorem 7.10 also $r(\mathcal{A})=1$, again a contradiction. Hence there exists a minor of order 2 which is zeroless. This means that $\operatorname{mr}(\mathcal{A}) \geq 2$. Combining, we obtain that $\operatorname{mr}(\mathcal{A})=2$.

\section{Other approaches to error analysis in matrix calculus}

Our approach to error analysis of matrices is characterized by treating, at every entry, an error as a set of numbers around a specific value, resulting in a rather strong algebraic structure for error propagation, to which basic notions of linear algebra can be adapted. In this section we intend to situate this approach with respect to existing methods, in particular classical asymptotic theory, Van der Corput's neutrices of functions, interval calculus, parametrization and probabilistic methods.

First we note that due to the Sorites property $[12,13,37]$ of neutrices, we tend to model imprecisions (say, coming from measuring and rounding off) more than uncertainties, which may have other sources, like imperfect models in the case of simplifications of too complex reality, or the impossibility to take into account intrinsic stochastic aspects [34]. Also our approach is theoretical, and aims at a description of the behavior of errors. In concrete situations it must be interpreted before it can be implemented in numerical analysis and computer calculations; what is small, what can be neglected?

We share these problems of interpretation with common asymptotics based on neglection of Oh's and oh's, which have been defined in terms of groups of functions in [3], and Van der Corput's neutrix theory [5], where also other groups of functions (for instance oscillatory functions) may be neglected. In these settings algebraic operations are well-defined, but they do not lead to structures as strong as a Complete Arithmetical Solid. For example, a set of functions in general does not allow for an order relation. Also, due the functional dependence there are serious complications when trying to handle multiple errors individually, and it seems that there exist no thorough applications to the propagation of errors in linear algebra or matrix calculus.

Common error analysis models errors more or less informally as small intervals around a value, resulting from a measurement or an estimation [35]. This enables individual treatment of errors and algebraic operations on them, which have essentially the same form as the Minkowski operations of Definition 2.2. The informal nature of error analysis inhibits the development of a strong algebraic calculus and the formulation of the basic notions of linear algebra. On the other hand, the implementation as an interval is obvious, though discussion is possible on the interpretation of "small". Proper interval calculus $[1,14,27,30]$ is part of formal mathematics, and stronger algebraic properties hold for operations. However it is no longer built on the Minkowski operations of Definition 2.2, and due to problems of subdistributivity and intersection not in all cases simple laws can be given, moreover the algebraic operations do not need to respect order. Interval analysis of matrix operations has been studied [30], though not from the point of view of algebraic properties. Of all the approaches the implementation of interval calculus is perhaps the most straightforward.

Methods of attributing to imprecise factors one or more parameters taking values in definite intervals, have been proposed for, in particular, linear programming. Multiparameter methods enable individual treatment of errors and have been proposed by among others Gass and Saaty [19, 20] and Nedoma and Gall [17]. By their functional nature the implementation is straightforward, and their numerical implications are intensively studied in e.g. [6]. Though one of the issues is the study of degeneracy [17], it has been recognized that excessive complications seem to avoid the development of a thorough algebraic theory.

Fuzzy set theory $[31,38]$ treats uncertainties and imprecisions by dealing with sets in the form of representative functions other than characteristic functions. By nature this approach does not address the Sorites 
property, but permits individual treatment of errors. The method has been applied to matrix calculus. Operations are clearly defined, and have been studied from the numerical point of view [31]. There does not seem to exist a strong algebraic theory of matrix computations, including the basic notions of linear algebra.

There is a large variety of statistic and stochastic approaches to the analysis of errors [4, 23,33], and they are used to study uncertainties and imprecisions of several kinds, also within matrix calculus [7,16, 28]. Computer simulations facilitate their implementation, but the establishment of a theory of linear algebra in the setting of error propagation with individual errors again seems to be complicated by the fact that probability distributions are functional, thus behaving less appropriately under algebraic operations.

Summarizing, we defend that the approach by external numbers respects the imprecision of errors, while allowing for a calculus for error propagation of moderate complexity, which yields insights at an intermediate level between qualitative and quantitative analysis. This calculus has stronger algebraic properties than other approaches, which however are mostly easier to implement.

\section{References}

[1] G. Alefeld, G. Mayer, Interval analysis: theory and applications, Journal of Computational and Applied Mathematics, 2000, 121, 421-464.

[2] I. P. van den Berg, A decomposition theorem for neutrices, Annals of Pure and Applied Logic, 2010, 161, 851-865.

[3] N. G. de Bruijn, Asymptotic analysis, 1961, North-Holland Pub. Co., Amsterdam.

[4] A. A. Clifford, Multivariate error analysis: a handbook of error propagation and calculation in many-parameter systems, 1973, Wiley, New York.

[5] J. G. van der Corput, Introduction to the neutrix calculus, Journal d'Analyse Mathématique, 1958, 7, 291-398.

[6] V. M. Charitopoulos, L. G. Papageorgiou, V. Dua, Multi-parametric linear programming under global uncertainty, AIChE J, 2017, 63(9), 3871-3895.

[7] T. Dayar, J. Fourneau, N. Pekergin, Transforming stochastic matrices for stochastics comparison with the st-order, RAIRO Oper. Res., 2003, 37, 85-97.

[8] F. Diener, M. Diener, (Ed.), Nonstandard analysis in practice, 1995, Springer-Verlag, Berlin.

[9] F. Diener, G. Reeb, Analyse nonstandard, 1989, Hermann, Paris.

[10] B. Dinis, I. P. van den Berg, Algebraic properties of external numbers, Journal of Logic \& Analysis, 2011, 3(9), 1-30.

[11] B. Dinis, I. P. van den Berg, Axiomatics for the external numbers of nonstandard analysis, Journal of Logic \& Analysis, 2017, 9(7) 1-47.

[12] B. Dinis, I. P. van den Berg, Neutrices and External Numbers: A Flexible Number System, 2019, Chapman \& Hall.

[13] H. Dominic, R. Diana, Sorites Paradox, The Stanford Encyclopedia of Philosophy, Summer 2018 Edition, https://plato. stanford.edu/archives/sum2018/entries/sorites-paradox/

[14] V. Gabrel, C. Murat, N. Remli, Linear programming with interval right hand-sides, International Transactions in Operations Research, 2010, 17, 397-408.

[15] W. Gähler, S. Gähler, Contributions to fuzzy analysis, Fuzzy Sets and Systems, 1999, 105, 201-224.

[16] P. A . Gagniuc, Markov Chains: From theory to implementation and experimentation, 2017, Wiley, New York.

[17] T. Gal, J. Nedoma, Multiparametric linear programming, Management Science, 1972, 18, 406-422.

[18] F. R. Gantmacher, The theory of matrices, vols I, and II, 1960, Chelsea Publishing Co., New York.

[19] S. I. Gass, T. L. Saaty, Parametric objective function (Part 1), Journal of the Operations Research Society of America, 1954, 2 , 316-319.

[20] S. I. Gass, T. L. Saaty, Parametric objective function (Part 2), Journal of the Operations Research Society of America, 1955, 3, 395-401.

[21] J. Justino, I. P. van den Berg, Cramer's rule applied to flexible systems of linear equations, Electronic Journal of Linear Algebra, 2012, 24, 126-152.

[22] J. Justino, Nonstandard linear algebra with error analysis (Ph.D thesis), 2013, Évora: University of Évora.

[23] P. Kall, J. Mayer, Stochastic linear programming. Models, theory, and computation, 2011, Springer US, Boston.

[24] V. Kanovei, M. Reeken, Nonstandard analysis, axiomatically, 2004, Springer-Verlag, Berlin.

[25] F. Koudjeti, I. P. van den Berg, Neutrices, external numbers and external calculus In: F. and M. Diener (Ed.) Nonstandard analysis in practice, 1995, Springer-Verlag, Berlin.

[26] W. Lyantse, T. Kudryk, Introduction to nonstandard analysis, 1997, VNTL Publishers, Lviv.

[27] R. E. Moore, R. Baker Kearfott, M. J. Cloud, Introduction to interval analysis, 2009, SIAM, Philadelphia.

[28] S. Natarajan, T. Raghavan, K. Viswanath, On stochastic matrices and kernels, Theory of Probability and Its Applications, $1967,12,337-341$. 
[29] E. Nelson, Internal set theory: A new approach to nonstandard analysis, Bulletin of the American Mathematical Society, 1977, 83, 1165-1198.

[30] A. Neumaier, Interval methods for systems of equations, 1991, Cambridge University Press.

[31] M. Otadi, M. Mosleh, Solving fully fuzzy matrix equations, Applied Mathematical Modelling, 2012, 36(12), 6114-6121.

[32] M. Petrich, N. Reilly, Completely regular semigroups, 1999, Canadian Mathematical Society Series of Monographs and Advanced Texts, Wiley, New York.

[33] A. Saltelli, M. Ratto, T. Andres, F. Campolongo, J. Cariboni, D. Gatelli, M. Saisana, S. Tarantola, Global sensitivity analysis, 2008, The Primer. Wiley, West Sussex.

[34] P. Smets, Imperfect Information: Imprecision and Uncertainty In: A. Motro (Ed.) Uncertainty Management in Information Systems, 1997, Springer, Boston, MA.

[35] J. R. Taylor, An introduction to error analysis: The study of uncertainties in physical measurements (2nd ed.), 1997, University Science Books.

[36] N. V. Tran, I. P. van den Berg, A parameter method for linear algebra and optimization with uncertainties, Optimization, 2019, DOI: $10.1080 / 02331934.2019 .1638387$.

[37] S. E. Weiss, The sorites fallacy: What difference does a peanut make?, Synthese, 1976, 33, 253-272, URL: http://www.jstor.org/stable/20115132.

[38] M. J. Wierman, An introduction to the mathematics of uncertainty, 2010, Center for the mathematics of uncertainty, Creighton University. 